\title{
Abundances for globular cluster giants. I. Homogeneous metallicities for 24 clusters $^{\star}$
}

\author{
E. Carretta ${ }^{1,3}$ and R.G. Gratton ${ }^{2}$ \\ 1 Osservatorio Astronomico di Bologna, Via Zamboni 33, I-40126 Bologna, Italy \\ 2 Osservatorio Astronomico di Padova, Vicolo dell'Osservatorio 5, I-35122 Padova, Italy \\ 3 Dipartimento d'Astronomia, Università di Padova, Vicolo dell'Osservatorio 5, I-35122 Padova, Italy
}

Received February 28; accepted May 21, 1996

\begin{abstract}
We have obtained high resolution, high signalto-noise ratio CCD echelle spectra of 10 bright red giants in 3 globular clusters (47 Tuc, NGC 6752 and NGC 6397) roughly spanning the whole range of metallicities of the galactic globular cluster system. The analysis of this newly acquired material reveals no significant evidence of starto-star variation of the $[\mathrm{Fe} / \mathrm{H}]$ ratio in these three clusters. Moreover, a large set of high quality literature data (equivalent widths from high dispersion CCD spectra) was reanalyzed in an homogeneous and self-consistent way to integrate our observations and derive new metal abundances for more than 160 bright red giants in 24 globular clusters (i.e. about $16 \%$ of the known population of galactic globulars). This set was then used to define a new metallicity scale for globular clusters which is the result of high quality, direct spectroscopic data, of new and updated model atmospheres from the grid of Kurucz (1992), and of a careful fine abundance analysis; this last, in turn, is based on a common set of both atomic and atmospheric parameters for all the stars examined. Given the very high degree of internal homogeneity, our new scale supersedes the offsets and discrepancies existing in previous attempts to obtain a metallicity scale. The internal uncertainty in $[\mathrm{Fe} / \mathrm{H}]$ is very small: 0.06 dex (24 clusters) on average, and can be interpreted also as the mean precision of the cluster ranking. Compared to our system, metallicities on the widely used Zinn and West's scale are about 0.10 dex higher for $[\mathrm{Fe} / \mathrm{H}]>-1,0.23$ dex lower for $-1<[\mathrm{Fe} / \mathrm{H}]<-1.9$ and 0.11 dex too high for $[\mathrm{Fe} / \mathrm{H}]<-1.9$. The non-linearity of the Zinn and West's scale is significant even at $3 \sigma$ level. A quadratic transformation is given to correct older values to the new scale in the range of our calibrating clusters $\left(-2.24 \leq[\mathrm{Fe} / \mathrm{H}]_{\mathrm{ZW}} \leq-0.51\right)$. A minor disagreement is found at low metallicities between the metallicity scale based on field and cluster RR Lyrae variables (via a new
\end{abstract}

Send offprint requests to: E. Carretta

* Table 9 also available in electronic form at the CDS via anonymous ftp 130.79 .128 .5 or via http://cdsweb.ustrabg.fr/Abstract.html calibration of the $\Delta S$ index) and our new cluster metallicities. It could be tentatively ascribed to non-linearity in the $[\mathrm{Fe} / \mathrm{H}]-\Delta S$ relationship. The impact of new metallicities on major astrophysical problems is exemplified through a simple exercise on the Oosterhoff effect in the classical pair M 3 and M 15.

Key words: stars: abundances — stars: population II globular clusters: general

\section{Introduction}

It is widely recognized that globular clusters (GCs) are cornerstones for the solution of a large variety of problems concerning the formation and evolution of galaxies. They are among the oldest objects formed in the Galaxy and, as a consequence, can be used as tracers of the early chemical evolutionary phases of the galactic environment. In principle, as a first guess, one can consider the total metal abundance (traditionally indicated by the ratio $[\mathrm{Fe} / \mathrm{H}]^{1}$ ) of a globular cluster as representative of the original composition of the gas from which it formed, and the variations in $[\mathrm{Fe} / \mathrm{H}]$ among clusters as a fossil recording of the chemical enrichment history occurred in the Galaxy. It is nowadays well known, however, that this simple view is complicated by other phenomena: the chemical composition of the atmospheres of stars can be altered by processes occurring during their evolution (see Smith 1987, and Kraft 1994 for reviews). Even if the actual mechanisms are not completely understood yet, it seems that surface abundances of only the lightest element $(\mathrm{C}, \mathrm{N}, \mathrm{O}, \mathrm{Na}$, and $\mathrm{Al})$ are affected, while $\mathrm{Fe}$ abundances are unchanged. In a following paper we will discuss in detail this aspect.

1 We adopt the usual spectroscopic notation, i.e. $[X]=$ $\log (X)_{\text {star }}-\log (X) \odot$ for any abundance quantity $\mathrm{X}$, and $\log$ $\epsilon(X)=\log \left(N_{\mathrm{X}} / N_{\mathrm{H}}\right)+12.0$ for absolute number density abundances. 
In the present paper we explore the possibility of building a new metallicity scale for galactic globular clusters using only $[\mathrm{Fe} / \mathrm{H}]$ values obtained from fine analysis of high dispersion spectra. Although globular cluster distances restrict applicability of this technique to the brightest giants, it provides direct accurate and quantitative determination of the actual metallicity of stellar atmospheres.

It is rather surprising that despite the advent of CCD detectors and of sophisticated and efficient spectrographs, the increasing number of measures of cluster metallicities has been (and is!) almost totally ignored in a variety of astrophysical problems involving this parameter. The most widely used metallicity scale for globular clusters is in fact the one obtained by Zinn \& West (1984; hereinafter ZW) and Zinn (1985) from a calibration of integrated parameters of globular clusters. The main advantage of using integrated parameters is that they can be easily measured even for the most distant objects in the Galaxy: homogeneous results can then be obtained for almost all known galactic clusters. However, integrated parameters are not directly related to metal abundances, and their use as abundance indices requires an accurate calibration in terms of the actual content of $[\mathrm{Fe} / \mathrm{H}]$. Reflecting uncertainties present at that epoch in abundances from high dispersion spectra, ZW attributed very low weight to direct abundance determinations for globular cluster stars when they constructed their metallicity scale for globular clusters.

Since ZW work, a number of accurate high-resolution spectroscopic determinations of metal abundances for stars in globular clusters appeared in the literature. However, only the work of Gratton and coworkers (Gratton et al. 1986; Gratton 1987; Gratton \& Ortolani 1989: G86, G87, G89 respectively; collectively G8689) was aimed at a systematic determination of abundances for a large number of clusters (spectra for giants in 17 clusters were actually analyzed). However, since completion of the Gratton and coworkers survey, there have been significant progresses both in the analysis techniques and in observing facilities. In fact the new Kurucz (1992, hereinafter K92) model atmospheres allow an homogeneous comparison between solar and stellar abundances, a major drawback of former analysis of abundances for globular cluster stars (see e.g., Leep et al. 1987). Furthermore, improvements in high resolution spectrographs and detectors allow better spectra to be obtained for a larger number of stars: a major contribution has been done by observations with the Hamilton spectrograph at Lick by Kraft, Sneden \& coworkers (Sneden et al. 1991; Kraft et al. 1992; Sneden et al. 1992; Kraft et al. 1993; Sneden et al. 1994; Kraft et al. 1995; hereinafter, SKPL1, SKPL2, SKPL3, SKPL4, SKPL5, SKPL6 and SKPL on the whole, for brevity). Regretfully, a homogeneous abundance scale based on highresolution spectroscopic data does not exist yet, mainly due to inconsistencies in the model atmospheres and in the atomic parameters adopted in the various investiga- tions. Moreover, the need for such an improved scale is continuously growing, due to the high degree of accuracy required by a variety of problems, one for all: the longdebated calibration of the absolute magnitude of the horizontal branch in terms of the metal abundance. Minor variations in the adopted metallicities could result, ultimately, in non-trivial changes in globular clusters ages.

Our goal is to exploit recent observational and theoretical progresses to construct a new, reliable metallicity scale for globular clusters, completely based on highquality data for red giant stars in 24 clusters. To this purpose, we obtained new data for a few stars in three southern clusters using the Long Camera mounted on the ESO CASPEC spectrograph, and reanalyzed published equivalent widths $(E W \mathrm{~s})$, mainly from the Gratton and coworkers and Lick surveys, integrated by other sources of similar quality for clusters not included in those studies. These data were analyzed in a totally self-consistent way, allowing a modern calibration of the abundance indices considered by ZW.

In Sect. 2 we present the new data, and in Sect. 3 the data adopted from literature. In Sects. 4 and 5 we discuss respectively the atmospheric and atomic parameters required for the abundance analysis. Our results will be exposed in Sect. 6, together with a discussion of the error sources. After a comparison with previous works (Sect. 7), we present our conclusions in Sect. 8.

Table 1. Observed spectral regions

$$
\lambda_{\mathrm{c}}(\AA) \quad \text { Spectral interv. Indicators }
$$

\begin{tabular}{lll}
\hline 4200 & $3800-4600$ & CH $(G$ band $)$ \\
5100 & $4700-5480$ & $\mathrm{C}_{2}$ \\
6300 & $5950-6700$ & {$[\mathrm{O}$ I] red doublet } \\
8000 & $7750-8380$ & CN red system
\end{tabular}

\section{Observational data}

We selected a sample of 10 red giant branch (RGB) stars in $3 \mathrm{GCs}$, representative of the typical range of metal abundance of these objects, i.e. 47 Tuc (high metallicity, $[\mathrm{Fe} / \mathrm{H}] \simeq-0.8$ dex, 3 stars), NGC 6752 (intermediate metallicity, $[\mathrm{Fe} / \mathrm{H}] \simeq-1.5,4$ stars) and NGC 6397 (low metallicity, $[\mathrm{Fe} / \mathrm{H}] \simeq-2.0,3$ stars).

Due to obvious flux limitations, we restricted our observations to the brightest globular cluster stars to obtain observational material good enough (i.e. adequately high resolution and $S / N$ ratios) for fine abundance analysis 
Table 2. Program stars observed in 47 Tuc, NGC 6397 and NGC 6752

\begin{tabular}{lllll}
\hline \hline Cluster & Star & $V$ & $B-V$ & $(V-K)_{0}$ \\
& & & & \\
\hline \multirow{2}{*}{ TUC } & 5422 & 12.47 & 1.40 & 3.23 \\
& 5529 & 11.87 & 1.59 & 3.86 \\
NGC 6397 & C25 & 12.22 & 0.96 & 2.29 \\
& C211 & 10.16 & 1.46 & 3.13 \\
& C428 & 11.50 & 1.05 & 2.47 \\
NGC 6752 & A31 & 10.80 & 1.60 & 3.69 \\
& A45 & 11.57 & 1.23 & 3.00 \\
& A61 & 11.71 & 1.13 & 2.91 \\
& C9 & 12.37 & 1.03 & 2.62 \\
& & & & \\
\hline
\end{tabular}

References - 47 Tuc: Frogel et al. (1981); NGC 6397 and NGC 6752: Frogel et al. (1983).

with reasonable exposure times. All observed stars were brighter than $V=12.5$; the faintest giant observed, star 5422 in 47 Tuc, has $M_{V}^{0}=-0.96$ (adopting a true distance modulus of 13.31 and $A_{V}=0.12$ from Djorgovski 1993). Moreover, stars were selected to have infrared photometry available, in particular in the $K$ band, for an accurate determination of the effective temperature $T_{\text {eff }}$.

The observational material was acquired in two runs: October 1990 and June 1991. In both runs, echelle spectra of the program stars were obtained with the CASPEC spectrograph (in the Long Camera configuration) at the $3.6 \mathrm{~m}$ ESO telescope at La Silla, using a 31.6 lines $/ \mathrm{mm}$ echelle grating. The slit width was adjusted in order to give resolution $R \sim 30000$.

We tried to obtain a spectral coverage as large as possible, to observe spectral features of different atomic and molecular species and to compare abundances of the same element as derived from different indicators. In Table 1 we list the main features (abundance indicators) of the spectral regions observed. Table 2 lists the literature photometric measurements of the program stars, and Table 3 the observed intervals for each star.

Exposure times ranged from 15 to 70 minutes to reduce cosmic ray contamination; we usually tried to obtain more than one spectrum for each object to eliminate the spurious events. A quartz lamp (for flat fielding) and a Thorium-Argon lamp (for wavelength calibration) were acquired after each program star exposure, with the telescope at the same position of the program star exposures. Besides, fast-rotating early-type stars were observed each night to remove telluric lines (see below). Bias frames have been taken at the beginning of each night to account for readout noise.
Table 3. Spectral intervals observed for the program stars

\begin{tabular}{llrrr}
\hline \hline Cluster & Star & $\begin{array}{c}\lambda_{\mathrm{C}} \\
(\AA)\end{array}$ & $\begin{array}{r}t_{\text {exp }} \\
(\min .)\end{array}$ & $S / N$ \\
& & & & \\
\hline \multirow{3}{*}{ Tuc } & 5422 & 5100 & 240 & 145 \\
& & 6300 & 100 & 111 \\
& & 8000 & 59 & 45 \\
& 5529 & 4200 & 70 & 60 \\
& & 5100 & 70 & 100 \\
& & 6300 & 35 & 56 \\
& & 8000 & 120 & 100 \\
& 8406 & 5100 & 240 & 120 \\
NGC 6397 & & 6300 & 90 & 107 \\
& C25 & 6300 & 80 & 88 \\
& C211 & 4200 & 40 & 50 \\
& & 6300 & 15 & 117 \\
& C428 & 6300 & 41 & 91 \\
NGC 6752 & A31 & 6300 & 30 & 130 \\
& A45 & 6300 & 45 & 105 \\
& A61 & 6300 & 50 & 101 \\
& C9 & 6300 & 90 & 104 \\
& & & & \\
\hline & & & &
\end{tabular}

\subsection{Data analysis and equivalent widths}

The first steps in CCD reduction (bias subtraction, echelle order identification, scattered light subtraction, order extraction and wavelength calibration) were performed using standard packages implemented in $\mathrm{IRAF}^{2}$ environment. Off-order scattered light was eliminated through bi-dimensional fitting along the dispersion and in the orthogonal direction. The spectra were then wavelength calibrated using a dispersion solution in two dimensions, derived from the Th-Ar lamps taken after each spectrum, and one-dimensional spectra were extracted using an $o p$ timal extraction algorithm implemented in the package.

The next steps of the analysis were then performed using the ISA package (Gratton 1988), purposely developed to deal with high-resolution spectra.

The blaze function was taken into account by dividing the spectra by dome flat fields; the continuum was then traced on each individual spectrum for every object. Whenever we had multiple exposures of the same star, spurious events and spikes due to cosmic rays were eliminated comparing different spectra. We then used the spectra of featureless, rapidly rotating, early-type stars for accurate removal of the telluric $\mathrm{O}_{2}$ features, affecting in particular the $6300 \AA$ region. We first identified

2 IRAF is distributed by the National Optical Astronomy Observatories, which is operated by the Association of Universities for Research in Astronomy, Inc., under cooperative agreement with the National Science Foundation. 
atmospheric features in the solar spectrum then we measured each line in the spectra of early-type stars acquired at different airmasses $z$. From these measurements we derived a mean relationship between the $E W \mathrm{~s}$ and $z$. For each star a synthetic spectrum of the atmospheric lines was then computed and convolved with the instrumental profile; $\mathrm{O}_{2}$ features were finally cancelled out by dividing the spectrum of each program star by the appropriate synthetic spectrum. This procedure allows to correct each star for the appropriate airmass; moreover it does not introduce additional noise in the object spectra.

The resulting cleaned spectra were then added to improve the $S / N$, after correcting for the change in the (geocentric) radial velocity of the star. Finally, a new continuum was traced. Figure 1 shows the tracing of a portion of our co-added and normalized CASPEC spectra for two program stars.

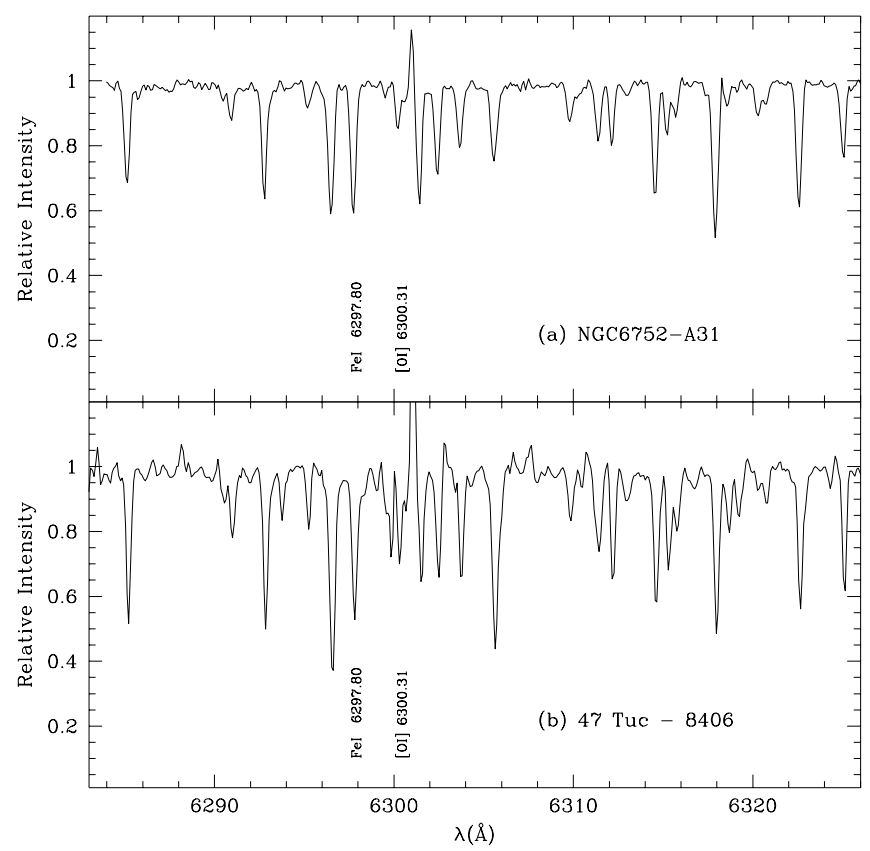

Fig. 1. Tracing of a portion of the co-added, normalized spectra of 2 stars in the $6300 \AA$ region

Final available spectra are listed in Table 3 . We note that the following abundance analysis uses only $E W$ s measured in the spectral regions centered at 5100 and $6300 \AA$. The main reason is that they are less affected by telluric bands and richer of stellar features (as compared to the $8000 \AA$ region), and there is less concern related to line crowding and continuum level identification (as compared to the $4200 \AA$ region); this last feature is of particular importance for the the spectra of stars in the metal-rich cluster 47 Tuc.

Equivalent widhts of various element were then measured in the two mentioned regions: in the following we will refer only to iron lines. Gaussian profiles were fitted to the observed profiles; when the number of clean lines was very low (e.g., for Fe II lines), we first derived a relationship between $E W$ and central depth $r_{\mathrm{C}}$ from unblended lines and then we used it to add some new EW's for measured $r_{\mathrm{C}}$ 's. The number of measured lines depends on $S / N$ and on the star metallicity; generally, some $25-50$ Fe lines were measured for NGC 6397 stars, some 50-70 for NGC 6752 stars and from 50 to 100 for 47 Tuc stars. In the following analysis, only lines with $E W>10 \mathrm{~m} \AA$ were used. Line parameters (see Sect. 5) and $E W$ s are listed in Table 4, both for Fe I and Fe II lines.

We have 3 stars (namely, 47 Tuc-5529, NGC 6397-C211 and NGC 6752-A45) in common with another recent, high dispersion analysis by Norris and $\mathrm{Da}$ Costa (1995; hereinafter, NDC), at about the same resolution we used: this allows a comparison between the two sets of $E W \mathrm{~s}$, which is shown in Fig. 2. The average difference is ${ }^{3}: E W_{\mathrm{CG} 96}-E W_{\mathrm{NDC}}=6.1 \pm 1.1 \mathrm{~m} \AA(\sigma=9.2 \mathrm{~m} \AA$, 76 lines $\left.^{4}\right)$. Note that here we regard this comparison as an external check of the accuracy of our $E W$ 's measurements, but since we used data from NDC to enlarge the sample of analyzed stars (see below), the above comparison has to be regarded also as a self-consistency test on our total set of $E W \mathrm{~s}$.

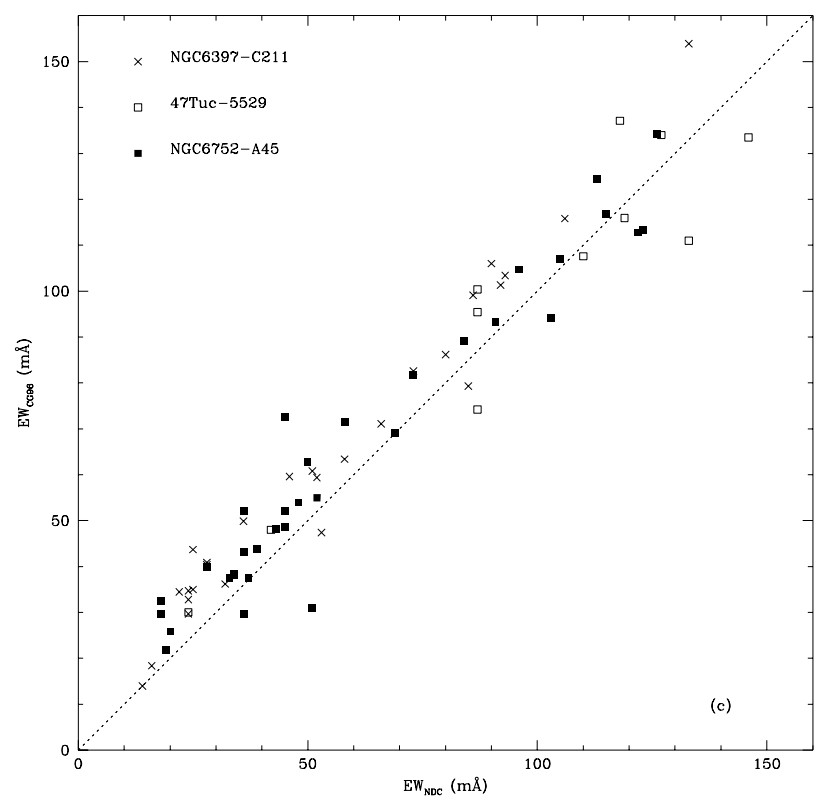

Fig. 2. Comparison of our $E W \mathrm{~s}$ with those of NDC for the 3 stars in common

3 We will indicate with the subscript CG96 the sample of our observed program stars and simply CG the results obtained using the whole sample (observations plus literature data) analyzed in the present paper.

4 Throughout this paper, the symbol $\sigma$ will indicate the standard deviation of a single measurement, while the value after the symbol \pm will refer to the standard deviation of the mean. 
Table 4. Equivalent widths for giants in 47 Tuc, NGC 6397 and NGC 6752

\begin{tabular}{|c|c|c|c|c|c|c|c|c|c|c|c|c|c|}
\hline Element & $\lambda(\AA)$ & E.P. & $\log g f$ & $\begin{array}{r}47 \mathrm{Tuc} \\
5422 \\
\mathrm{EW}\end{array}$ & $\begin{array}{r}47 \mathrm{Tuc} \\
5529 \\
\mathrm{EW}\end{array}$ & $\begin{array}{r}47 \mathrm{Tuc} \\
8406 \\
\mathrm{EW}\end{array}$ & $\begin{array}{r}\text { NGC6397 } \\
25 \\
\mathrm{EW}\end{array}$ & $\begin{array}{r}\text { NGC6397 } \\
\text { C211 } \\
\text { EW }\end{array}$ & $\begin{array}{r}\text { NGC6397 } \\
\text { C428 } \\
\text { EW }\end{array}$ & $\begin{array}{r}\text { NGC6752 } \\
\text { A31 } \\
\text { EW }\end{array}$ & $\begin{array}{r}\text { NGC6752 } \\
\text { A45 } \\
\text { EW }\end{array}$ & $\begin{array}{r}\text { NGC6752 } \\
\text { A61 } \\
\text { EW }\end{array}$ & $\begin{array}{r}\text { NGC6752 } \\
\mathrm{C} 9 \\
\mathrm{EW}\end{array}$ \\
\hline $\mathrm{Fe} \mathrm{I}$ & 5217.30 & 3.21 & -1.07 & 138.6 & 0.0 & 0.0 & 0.0 & 0.0 & 0.0 & 0.0 & 0.0 & 0.0 & 0.0 \\
\hline $\mathrm{Fe} \mathrm{I}$ & 5250.21 & 0.12 & -4.90 & 0.0 & 0.0 & 197.0 & 0.0 & 0.0 & 0.0 & 0.0 & 0.0 & 0.0 & 0.0 \\
\hline $\mathrm{Fe} \mathrm{I}$ & 5253.02 & 2.28 & -3.81 & 0.0 & 0.0 & 85.9 & 0.0 & 0.0 & 0.0 & 0.0 & 0.0 & 0.0 & 0.0 \\
\hline $\mathrm{Fe} \mathrm{I}$ & 5956.70 & 0.86 & -4.57 & 166.4 & 0.0 & 177.9 & 31.3 & 101.3 & 41.7 & 0.0 & 116.8 & 103.0 & 0.0 \\
\hline $\mathrm{Fe} \mathrm{I}$ & 5976.79 & 3.94 & -1.29 & 92.7 & 0.0 & 101.0 & 0.0 & 40.9 & 24.9 & 70.1 & 55.0 & 38.7 & 0.0 \\
\hline $\mathrm{Fe} \mathrm{I}$ & 5984.82 & 4.73 & -0.28 & 0.0 & 0.0 & 0.0 & 0.0 & 35.0 & 17.7 & 0.0 & 0.0 & 42.3 & 0.0 \\
\hline $\mathrm{Fe} \mathrm{I}$ & 6015.24 & 2.22 & -4.66 & 33.5 & 0.0 & 49.9 & 0.0 & 0.0 & 0.0 & 21.0 & 0.0 & 0.0 & 0.0 \\
\hline $\mathrm{Fe} \mathrm{I}$ & 6019.37 & 3.57 & -3.21 & 15.4 & 24.9 & 15.8 & 0.0 & 0.0 & 0.0 & 0.0 & 0.0 & 0.0 & 0.0 \\
\hline $\mathrm{Fe} \mathrm{I}$ & 6027.06 & 4.07 & -1.18 & 92.6 & 0.0 & 94.7 & 0.0 & 29.7 & 0.0 & 69.0 & 0.0 & 0.0 & 0.0 \\
\hline $\mathrm{Fe} \mathrm{I}$ & 6034.04 & 4.31 & -2.35 & 14.0 & 0.0 & 27.6 & 0.0 & 0.0 & 0.0 & 0.0 & 0.0 & 0.0 & 0.0 \\
\hline $\mathrm{Fe} \mathrm{I}$ & 6054.08 & 4.37 & -2.22 & 21.5 & 0.0 & 0.0 & 0.0 & 0.0 & 0.0 & 0.0 & 0.0 & 0.0 & 0.0 \\
\hline $\mathrm{Fe} \mathrm{I}$ & 6056.01 & 4.73 & -0.45 & 64.6 & 0.0 & 81.1 & 0.0 & 18.4 & 11.3 & 52.8 & 43.1 & 37.9 & 0.0 \\
\hline $\mathrm{Fe} \mathrm{I}$ & 6078.50 & 4.79 & -0.37 & 76.4 & 0.0 & 81.4 & 0.0 & 0.0 & 0.0 & 0.0 & 0.0 & 0.0 & 43.9 \\
\hline $\mathrm{Fe} \mathrm{I}$ & 6079.01 & 4.65 & -0.95 & 54.9 & 0.0 & 56.5 & 0.0 & 0.0 & 0.0 & 22.7 & 32.6 & 0.0 & 0.0 \\
\hline $\mathrm{Fe} \mathrm{I}$ & 6082.72 & 2.22 & -3.59 & 0.0 & 0.0 & 0.0 & 0.0 & 40.4 & 0.0 & 0.0 & 62.7 & 0.0 & 0.0 \\
\hline $\mathrm{Fe} \mathrm{I}$ & 6093.65 & 4.61 & -1.29 & 33.3 & 0.0 & 41.2 & 0.0 & 0.0 & 0.0 & 0.0 & 0.0 & 0.0 & 0.0 \\
\hline $\mathrm{Fe} \mathrm{I}$ & 6094.38 & 4.65 & -1.52 & 0.0 & 0.0 & 17.7 & 0.0 & 0.0 & 0.0 & 0.0 & 0.0 & 0.0 & 0.0 \\
\hline $\mathrm{Fe} \mathrm{I}$ & 6096.67 & 3.98 & -1.73 & 53.5 & 0.0 & 82.3 & 0.0 & 0.0 & 0.0 & 41.7 & 29.8 & 0.0 & 0.0 \\
\hline $\mathrm{Fe} \mathrm{I}$ & 6098.25 & 4.56 & -1.77 & 0.0 & 0.0 & 41.9 & 0.0 & 0.0 & 0.0 & 0.0 & 0.0 & 0.0 & 0.0 \\
\hline $\mathrm{Fe} \mathrm{I}$ & 6120.26 & 0.91 & -5.94 & 76.0 & 95.4 & 78.1 & 0.0 & 0.0 & 0.0 & 64.8 & 32.5 & 0.0 & 0.0 \\
\hline $\mathrm{Fe} \mathrm{I}$ & 6151.62 & 2.18 & -3.26 & 124.6 & 133.5 & 130.2 & 0.0 & 59.6 & 23.0 & 125.2 & 0.0 & 57.4 & 57.7 \\
\hline $\mathrm{Fe} \mathrm{I}$ & 6157.73 & 4.07 & -1.25 & 0.0 & 0.0 & 107.6 & 0.0 & 34.5 & 0.0 & 78.8 & 0.0 & 48.5 & 0.0 \\
\hline $\mathrm{Fe} \mathrm{I}$ & 6165.36 & 4.14 & -1.46 & 64.5 & 83.1 & 71.3 & 0.0 & 0.0 & 0.0 & 46.4 & 29.7 & 0.0 & 13.8 \\
\hline $\mathrm{Fe} \mathrm{I}$ & 6173.34 & 2.22 & -2.85 & 145.9 & 0.0 & 147.1 & 0.0 & 82.6 & 46.6 & 157.7 & 94.2 & 91.5 & 87.2 \\
\hline $\mathrm{Fe} \mathrm{I}$ & 6187.40 & 2.83 & -4.11 & 33.2 & 0.0 & 0.0 & 0.0 & 0.0 & 0.0 & 0.0 & 0.0 & 0.0 & 0.0 \\
\hline $\mathrm{Fe} \mathrm{I}$ & 6187.99 & 3.94 & -1.58 & 69.6 & 0.0 & 83.0 & 0.0 & 0.0 & 0.0 & 47.7 & 0.0 & 0.0 & 23.5 \\
\hline $\mathrm{Fe} \mathrm{I}$ & 6213.44 & 2.22 & -2.55 & 157.8 & 0.0 & 184.6 & 38.8 & 103.4 & 54.9 & 167.5 & 112.7 & 116.3 & 95.6 \\
\hline $\mathrm{Fe} \mathrm{I}$ & 6219.29 & 2.20 & -2.42 & 170.2 & 184.3 & 180.0 & 62.8 & 115.8 & 66.5 & 187.9 & 134.2 & 131.1 & 116.3 \\
\hline $\mathrm{Fe} \mathrm{I}$ & 6226.74 & 3.88 & -2.02 & 0.0 & 0.0 & 83.1 & 0.0 & 0.0 & 0.0 & 29.6 & 0.0 & 0.0 & 0.0 \\
\hline $\mathrm{Fe} \mathrm{I}$ & 6240.65 & 2.22 & -3.23 & 0.0 & 0.0 & 137.0 & 0.0 & 0.0 & 0.0 & 0.0 & 0.0 & 0.0 & 0.0 \\
\hline $\mathrm{Fe} \mathrm{I}$ & 6280.62 & 0.86 & -4.35 & 172.1 & 0.0 & 203.4 & 0.0 & 0.0 & 56.7 & 0.0 & 0.0 & 0.0 & 0.0 \\
\hline $\mathrm{Fe} \mathrm{I}$ & 6297.80 & 2.22 & -2.73 & 170.9 & 0.0 & 180.5 & 38.6 & 99.1 & 50.6 & 152.6 & 113.2 & 112.8 & 90.6 \\
\hline $\mathrm{Fe} \mathrm{I}$ & 6301.51 & 3.65 & -0.72 & 0.0 & 0.0 & 0.0 & 55.7 & 0.0 & 0.0 & 134.6 & 0.0 & 0.0 & 0.0 \\
\hline $\mathrm{Fe} \mathrm{I}$ & 6311.51 & 2.83 & -3.14 & 0.0 & 0.0 & 0.0 & 0.0 & 0.0 & 0.0 & 72.9 & 30.9 & 21.7 & 0.0 \\
\hline $\mathrm{Fe} \mathrm{I}$ & 6315.82 & 4.07 & -1.65 & 80.4 & 0.0 & 90.5 & 0.0 & 0.0 & 0.0 & 0.0 & 37.6 & 0.0 & 0.0 \\
\hline $\mathrm{Fe} \mathrm{I}$ & 6322.69 & 2.59 & -2.39 & 135.3 & 0.0 & 149.2 & 0.0 & 79.3 & 0.0 & 141.8 & 104.8 & 93.7 & 72.6 \\
\hline $\mathrm{Fe} \mathrm{I}$ & 6353.84 & 0.91 & -6.44 & 53.6 & 0.0 & 0.0 & 0.0 & 0.0 & 0.0 & 0.0 & 0.0 & 0.0 & 0.0 \\
\hline $\mathrm{Fe} \mathrm{I}$ & 6380.75 & 4.19 & -1.32 & 78.7 & 0.0 & 90.3 & 0.0 & 16.5 & 15.2 & 52.8 & 40.0 & 22.6 & 34.0 \\
\hline $\mathrm{Fe} \mathrm{I}$ & 6392.54 & 2.28 & -3.95 & 74.3 & 74.2 & 69.8 & 0.0 & 0.0 & 0.0 & 0.0 & 25.9 & 21.7 & 0.0 \\
\hline $\mathrm{Fe} \mathrm{I}$ & 6393.61 & 2.43 & -1.43 & 216.1 & 0.0 & 0.0 & 87.2 & 0.0 & 99.6 & 222.5 & 0.0 & 0.0 & 0.0 \\
\hline $\mathrm{Fe} \mathrm{I}$ & 6498.95 & 0.96 & -4.66 & 151.3 & 0.0 & 0.0 & 0.0 & 86.2 & 0.0 & 172.1 & 97.4 & 81.8 & 77.5 \\
\hline $\mathrm{Fe} \mathrm{I}$ & 6518.37 & 2.83 & -2.46 & 113.3 & 0.0 & 120.6 & 17.3 & 47.4 & 22.6 & 119.7 & 72.6 & 59.4 & 46.3 \\
\hline $\mathrm{Fe} \mathrm{I}$ & 6581.22 & 1.48 & -4.68 & 108.8 & 0.0 & 0.0 & 0.0 & 32.8 & 0.0 & 118.1 & 48.5 & 36.8 & 0.0 \\
\hline $\mathrm{Fe} \mathrm{I}$ & 6593.88 & 2.43 & -2.39 & 159.6 & 0.0 & 158.0 & 0.0 & 106.0 & 49.7 & 0.0 & 124.4 & 118.6 & 104.9 \\
\hline $\mathrm{Fe} \mathrm{I}$ & 6608.03 & 2.28 & -3.94 & 95.5 & 0.0 & 95.7 & 0.0 & 0.0 & 0.0 & 65.2 & 26.5 & 27.2 & 18.4 \\
\hline $\mathrm{Fe} \mathrm{I}$ & 6609.12 & 2.56 & -2.66 & 136.8 & 0.0 & 144.0 & 16.5 & 63.4 & 25.3 & 134.7 & 89.1 & 74.7 & 55.0 \\
\hline $\mathrm{Fe} \mathrm{I}$ & 6667.43 & 2.45 & -4.35 & 0.0 & 0.0 & 0.0 & 0.0 & 0.0 & 0.0 & 17.8 & 0.0 & 0.0 & 0.0 \\
\hline $\mathrm{Fe}$ II & 5264.81 & 3.23 & -3.21 & 43.7 & 0.0 & & 0.0 & 0.0 & 0.0 & 0.0 & 0.0 & 0.0 & 0.0 \\
\hline $\mathrm{Fe}$ II & 5991.36 & 3.15 & -3.55 & 0.0 & 0.0 & 0.0 & 15.0 & 19.3 & 0.0 & 29.5 & 29.6 & 30.4 & 0.0 \\
\hline $\mathrm{Fe}$ II & 6084.11 & 3.20 & -3.80 & 0.0 & 0.0 & 0.0 & 0.0 & 17.8 & 0.0 & 20.4 & 0.0 & 0.0 & 0.0 \\
\hline $\mathrm{Fe}$ II & 6149.25 & 3.89 & -2.73 & 0.0 & 0.0 & 0.0 & 0.0 & 14.0 & 0.0 & 0.0 & 0.0 & 30.9 & 0.0 \\
\hline $\mathrm{Fe}$ II & 6369.46 & 2.89 & -4.21 & 0.0 & 0.0 & 0.0 & 0.0 & 14.0 & 10.8 & 0.0 & 20.1 & 13.5 & 0.0 \\
\hline $\mathrm{Fe}$ II & 6432.68 & 2.89 & -3.58 & 0.0 & 30.0 & 30.0 & 16.7 & 43.7 & 25.7 & 0.0 & 38.1 & 0.0 & 34.3 \\
\hline $\mathrm{Fe}$ II & 6456.39 & 3.90 & -2.09 & 52.1 & 48.0 & 44.2 & 0.0 & 49.9 & 37.8 & 48.1 & 52.0 & 57.9 & 48.0 \\
\hline $\mathrm{Fe}$ II & 6516.09 & 2.89 & -3.38 & 0.0 & 0.0 & 45.9 & 0.0 & 0.0 & 45.3 & 49.2 & 48.3 & 57.0 & 38.6 \\
\hline
\end{tabular}

\section{Literature data}

To increase our sample and to obtain a more statistically significant basis for our discussion, we also analyzed in a homogeneous way the globular cluster giants studied in two recent surveys: G8689 (41 red giants in 17 globular clusters) and SKPL (82 giants in 7 clusters). To these sets, we also added a full re-analysis of 3 giants in NGC 2298 (from McWilliam et al. 1992; McW92), 18 stars studied by Minniti et al. (1993; M 93) in 8 clusters, and 8 stars in 2 clusters from NDC. Table 5 lists the whole sample of globular clusters red giants analyzed in the present paper, with the source for each subset of data.

We simply adopted published $E W \mathrm{~s}$ for all samples, since the observational material consists in high-resolution ( $R \sim 30000)$, high $S / N$ spectra, fairly comparable to our own spectra. However, for the G8689 sample we had to make some corrections to the published $E W \mathrm{~s}$, before using them for a new analysis. In fact, in G87 the authors themselves noted that their $E W$ s were systematically higher than Mäckle et al. (1975) EWs for $\alpha$ Boo (Arcturus). This is due to the lower resolution $(R \sim 15000)$ used by G8689: unnoticed lines blend with the measured ones, resulting in an overall overestimate of the $E W \mathrm{~s}$. We derived the following relation between G8689 $E W \mathrm{~s}\left(E W_{\mathrm{G}}\right)$ and Mäckle et al. (1975) $E W \mathrm{~s}\left(E W_{\mathrm{M}}\right)$ for $\alpha$ Boo :

$$
E W_{\mathrm{M}}=0.914( \pm 0.044) E W_{\mathrm{G}}-14.3( \pm 17.6) \mathrm{m} \AA
$$

Strictly, this correction applies only to $E W$ s measured on spectra having metallicities similar to that of Arcturus $([\mathrm{Fe} / \mathrm{H}] \sim-0.5)$; we expect smaller corrections for more metal-poor stars. To verify this point we considered the stars in common between G8689 and the present data (NGC 6397-C428 and NGC 6752-C9); in both cases there are 17 lines in common between the old and the new analyses. The mean difference between the $E W \mathrm{~s}$ of our new analysis $E W_{\text {new }}$ and those of G8689 for these two stars are:

$E W_{\text {new }}-E W_{\mathrm{G}}=-3.0 \pm 1.8 \mathrm{~m} \AA$ 
Table 5. Complete list of globular clusters analyzed

\begin{tabular}{|c|c|c|}
\hline $\mathrm{GC}$ & Nr.Stars & Reference \\
\hline \multirow{2}{*}{ NGC 104-47 Tuc } & 3 & CG96 \\
\hline & 2 & G8689 \\
\hline NGC 288 & 2 & G8689 \\
\hline NGC 362 & 2 & G8689 \\
\hline NGC 1904 & 2 & G8689 \\
\hline NGC 2298 & 3 & McW92 \\
\hline NGC 3201 & 3 & G8689 \\
\hline \multirow[t]{2}{*}{ NGC 4590-M 68} & 2 & G8689 \\
\hline & 2 & M93 \\
\hline \multirow[t]{2}{*}{ NGC 4833} & 2 & G8689 \\
\hline & 1 & M93 \\
\hline NGC 5272-M 3 & 10 & SKPL \\
\hline NGC 5897 & 2 & G8689 \\
\hline \multirow[t]{2}{*}{ NGC 5904-M 5} & 13 & SKPL \\
\hline & 3 & G8689 \\
\hline NGC 6121-M 4 & 3 & G8689 \\
\hline NGC 6144 & 1 & M93 \\
\hline \multirow[t]{2}{*}{ NGC 6254-M 10} & 2 & G8689 \\
\hline & 14 & SKPL \\
\hline NGC 6205-M 13 & 23 & SKPL \\
\hline NGC 6341-M 92 & 9 & SKPL \\
\hline NGC 6352 & 3 & G8689 \\
\hline NGC 6362 & 2 & G8689 \\
\hline \multirow{4}{*}{ NGC 6397} & 3 & CG96 \\
\hline & 3 & G8689 \\
\hline & 5 & M93 \\
\hline & 2 & $\mathrm{NDC}$ \\
\hline NGC 6656-M 22 & 3 & G8689 \\
\hline \multirow[t]{4}{*}{ NGC 6752} & 3 & G8689 \\
\hline & 4 & CG96 \\
\hline & 6 & $\mathrm{NDC}$ \\
\hline & 3 & M93 \\
\hline \multirow[t]{2}{*}{ NGC 6838-M 71} & 10 & SKPL \\
\hline & 3 & G8689 \\
\hline \multirow[t]{2}{*}{ NGC 7078-M 15} & 3 & SKPL \\
\hline & 2 & M93 \\
\hline NGC 7099-M 30 & 2 & M93 \\
\hline
\end{tabular}

( $\sigma=10.5 \mathrm{~m} \AA$, 34 lines). Had we applied first Eq. (1) to correct the G8689's EWs, this difference would have been:

$E W_{\text {new }}-E W_{\mathrm{G}, \mathrm{corr}}=15.3 \pm 1.8 \mathrm{~m} \AA$

( $\sigma=10.3 \mathrm{~m} \AA, 34$ lines), where $E W_{\mathrm{G}, \text { corr }}$ are G8689 $E W \mathrm{~s}$ after transformation using Eq. (1). The overall correction is $\sim 18 \mathrm{~m} \AA$. We then estimate that while Eq. (1) gives the appropriate corrections to G8689 $E W$ s for metallicities similar to that of Arcturus $([\mathrm{Fe} / \mathrm{H}] \sim-0.5)$, these corrections should reduce to $\sim 1 / 6$ of this amount for a metallicity in between those of NGC 6752 and NGC

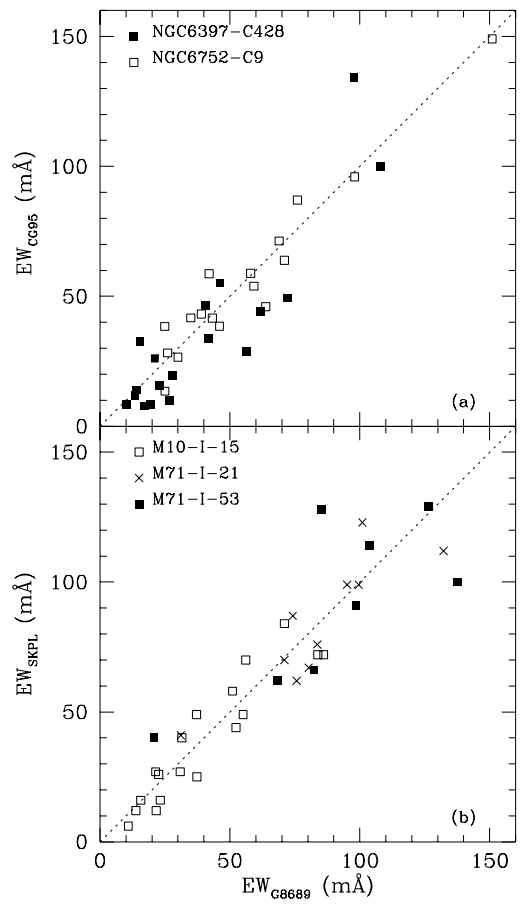

Fig. 3. a) Comparison between $E W$ s of G8689 sample after the correction and the $E W \mathrm{~s}$ of the present analysis (sample CG96) for NGC 6752-C9 and NGC 6397-C428; b) another test of self-consistency of our $E W \mathrm{~s}$, done by comparing the corrected $E W$ s from G8689 sample with those from SKPL sample for 3 stars in common between the two sets

$6397([\mathrm{Fe} / \mathrm{H}] \sim-1.7)$. We then included a metallicitydependent correction, obtaining:

$E W_{\mathrm{C}}=E W_{\mathrm{G}}-(0.7[\mathrm{Fe} / \mathrm{H}]+1.35)\left[(1-a) E W_{\mathrm{G}}-b\right]$

Equation (4) was then applied to the old $E W_{\mathrm{GS}}$ of G8689 to bring them to the same system of our higher resolution spectra. Figure 3 a compares $E W_{\mathrm{C}}$ with $E W_{\text {new }}$ for the two stars in common between the G8689 and the CG96 sample; the average difference is $E W_{\mathrm{C}}-E W_{\text {new }}=$ $3 \pm 2 \mathrm{~m} \AA$ ( $\sigma=10 \mathrm{~m} \AA, 34$ lines). The best fit line (with constant term put to zero) to the data in Fig. 3 a is: $E W_{\mathrm{C}}=$ $1.025 \pm 0.035 E W_{\text {new }}$, with a scatter of $11 \mathrm{~mA}$. In the following analisys, we used only lines with $E W_{\mathrm{C}}>20 \mathrm{~m} \AA$.

Figure $3 \mathrm{~b}$ shows another internal comparison, using G8689 (corrected) and SKPL $E W$ s for 3 stars: the agreement is very good, the average difference being $E W_{\text {SKPL }}-$ $E W_{\mathrm{G} 8689}=-0.5 \pm 2.4 \mathrm{~m} \AA$ ( $\sigma=14 \mathrm{~m} \AA, 36$ lines $)$. We estimated internal errors in $E W$ s of about $\pm 9 \mathrm{~m} \AA$ in G8689 set and $\pm 5 \mathrm{~m} \AA$ in CG96, SKPL and the other sets analyzed. We can then be confident that our results are based on an homogeneous and self-consistent dataset. 


\section{Atmospheric parameters}

Model atmospheres appropriate for each star were extracted from the grid of $\mathrm{K}_{2} 2^{5}$. Values of effective temperature $T_{\text {eff }}$ and surface gravity $\log g$ for giants observed in 47 Tuc, NGC 6752 and NGC 6397 (sample CG96) were taken from Frogel et al. (1981, 1983). Frogel et al's $T_{\text {eff's }}$ are based on visual-near infrared colours (mainly $V-K$ ), transformed into temperatures based on the Cohen et al. (1978; hereinafter CFP) scale. $\log g$ values are determined from the position of the stars in the colour-magnitude diagram (CMD). These values are considered as accurate as $\pm 100 \mathrm{~K}$ in $T_{\text {eff }}$ and \pm 0.3 dex in log $g$, including possible uncertainties in the effective temperature scale, as well in the adopted red giant mass, in the bolometric corrections and in the cluster distance modulus.

$T_{\text {eff }}$ and $\log g$ values for stars in the SKPL sample are also generally based on CFP's scale, except for giants in M 92 and M 15; in these two cases the authors used $T_{\text {eff }}$ 's from Carbon et al. (1982) and Trefzger et al. (1983). The reader should be warned that final values for $T_{\text {eff }}$ and $\log$ $g$, in the SKPL sample, are slightly different from the actual values on the CFP's scale. This is because SKPL adjusted the adopted photometric temperatures until a slope close to zero was achieved for the relationship between Fe I abundances from individual lines and excitation potential. However, as discussed in their papers, the agreement of photometric and final spectroscopic $T_{\text {eff }}$ was from the beginning well within the uncertainties of the temperature scale itself, so that neither the abundance ratios for individual stars nor the mean values for the clusters are significatively changed by adopting the tabulated values of atmospheric parameters from SKPL.

In the G8689 sample, $T_{\text {eff }}$ and $\log g$ values were homogeneously adopted from Frogel et al. $(1979,1981,1983)$ and are also used in the present work. For other data sets (McW92, NDC) we were able to use atmospheric parameters from Frogel and coworkers. Only half of the 18 stars from M 93 have $T_{\text {eff }}$ and $\log g$ values listed in Frogel et al.; for the missing stars, we used their original values, derived spectroscopically from the dependence of $T_{\text {eff }}$ on excitation potential and from the $\mathrm{Fe}$ ionization equilibrium. In fact, the parameters adopted by M 93 are with very good approximation on the CFP's scale: the mean

5 The model atmospheres considered in this paper are those in Kurucz's CD-ROM 13. In these models, convection description include an approximate consideration of possible overshooting in the stellar atmospheres. This point is discussed at length in Castelli et al. (1996), who conclude that at present is not possible to establish whether or not this treatment of convection should be preferred to a more traditional approach without any overshooting. However, this uncertainty is only of minor concern for the metallicity scale established in this paper, insofar the approach used for the Sun and the globular cluster giants is the same. for the stars in common are: $T_{\text {eff }}($ Frogel $)-T_{\text {eff }}(\mathrm{M} 93)=$ $-29 \pm 11 \mathrm{~K}(\sigma=30 \mathrm{~K}, 8$ stars $)$ and $\log g($ Frogel $)-\log g(\mathrm{M}$ $93)=-0.10 \pm 0.06 \operatorname{dex}(\sigma=0.17 \mathrm{dex}, 7 \mathrm{stars})$. For further details, see the original papers of G8689 and SKPL. The starting input values for $[\mathrm{Fe} / \mathrm{H}]$ were those from the original analyses.

Metallicities were obtained varying the metal abundance $[A / H]$ of the model until it was equal to the derived $[\mathrm{Fe} / \mathrm{H}]$ value. For the microturbulent velocity $v_{\mathrm{t}}$, the input values were changed until no trend in $\mathrm{Fe}$ abundance with the $E W$ of Fe I lines was present ${ }^{6}$. However, for giants in M 92 and M 15 only a few lines were available, so we took the values of $v_{\mathrm{t}}$ from the relationship:

$v_{\mathrm{t}}=-0.322( \pm 0.048) \cdot \log g+2.22( \pm 0.31)$

derived elsewhere (Carretta et al. 1996; Gratton \& Carretta 1996) for field stars. The adopted atmospheric parameters are listed in Table 9 (also available in electronic form) for all the stars studied in the present paper. Metallicities were obtained varying the metal abundance $[A / H]$ of the model until it was equal to the derived $[\mathrm{Fe} / \mathrm{H}]$ value.

\section{Oscillator strengths, line selection and solar abundances}

Only lines clean from blends on very high resolution solar spectra were considered in the analysis; for $\mathrm{Fe}$, the line list was extracted from Rutten \& van der Zalm (1984), and Blackwell et al. (1980). The adopted values for the oscillator strengths $g f \mathrm{~s}$ were determined following the same precepts of Clementini et al. (1995), who performed a high-dispersion study of the metal abundances of field RR Lyrae variables. Briefly, whenever possible laboratory $g f \mathrm{~s}$ were considered: for Fe I lines they were taken from papers of the Oxford group (for references, see Simmons \& Blackwell 1982) and Bard et al. (1991) and Bard \& Koch 1994), $g f$ s of the Oxford group being corrected upward to account for the systematic difference with those of Bard et al. (0.03 dex; see Clementini et al. 1995). $g f$ s for Fe II lines were taken from Heise \& Kock (1990), Biémont et al. (1991) and Hannaford et al. (1992). For lines lacking accurate laboratory determinations, $g f$ s were derived from an inverse solar analysis using the Holweger \& Müller (1974: HM) model atmosphere and the Fe abundance derived from the other lines. The adopted $g f$ values are also listed in Table 4; they can be integrated for other lines using $g f \mathrm{~s}$ in the larger line list of Clementini et al. (1995, Tables 3a, b).

In any differential analysis (i.e. a comparative analysis in which the zero point of the $[\mathrm{Fe} / \mathrm{H}]$ scale is set by the

6 Expected line strengths were used when determining the microturbulent velocity, following the prescription of Magain (1984). 
Sun) the assumed solar abundance is of paramount importance. The solar Fe abundance obtained with the set of $g f \mathrm{~s}$ described above and the K92 solar model atmosphere $(\log \epsilon(\mathrm{Fe})=7.52)$, taken as the reference value for the Sun throughout the present work, agrees with the meteoritic value of Anders \& Grevesse $(1989: \log \epsilon(\mathrm{Fe})=7.51)$. It is also very close to the value obtained using the HM model atmosphere $(\log \epsilon(\mathrm{Fe})=7.56$ : Castelli et al. 1996). A very similar value is obtained using Fe II lines when adopting the K92 model. The trend with excitation potential is also very small in the Sun.

We wish to stress here the importance of the consistency between these various determinations of the solar Fe abundances: in fact, the whole scale of metal abundance previously determined for globular clusters was uncertain, due to the rather large difference $(\sim 0.15$ dex $)$ between the solar Fe abundances obtained using the HM and the Bell et al. (1976: hereinafter BEGN) model atmospheres generally adopted in the analysis of cool cluster giants (see e.g., Leep et al. 1987). In fact, it was not clear what solar Fe abundance to use: either the one determined using a model extracted from the same grid used for cluster giants, or that obtained using the best solar model. Use of K92 atmospheres solves this problem, since we may now use models for giants extracted from the same grid which gives a solar $\mathrm{Fe}$ abundance in agreement with the best photospheric and meteoritic determinations.

Finally, we note that this choice of lines and of $g f$ values allows a direct comparison with the metallicity scale for the low-dispersion index $\Delta S$ in RR Lyrae stars derived by Clementini et al. (1995).

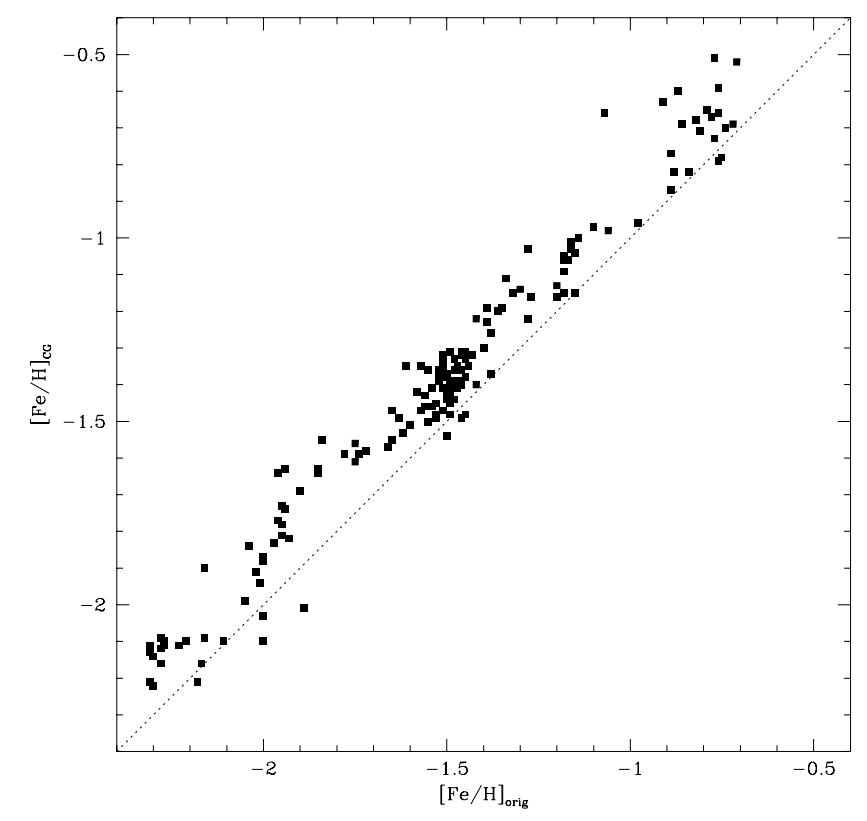

Fig. 4. Comparison of new $[\mathrm{Fe} / \mathrm{H}]$ values with those from the original analyses. Different symbols represent stars of different samples studied

\section{Results and error estimates}

Our Fe abundances for both the original program stars and for all reanalyzed stars are listed in Table 9 (last 7 columns) ${ }^{7}$. Final $[\mathrm{Fe} / \mathrm{H}]$ values adopted (last column) are those derived from neutral lines alone, since the number of Fe II lines with accurate $E W \mathrm{~s}$ was often too small. When individual clusters are considered, our $[\mathrm{Fe} / \mathrm{H}]$ values do not show any trend with $T_{\text {eff }}$ on the whole range $3800-4900 \mathrm{~K}$ (which approximatively corresponds to a range of about 2.5 in $\left.M_{\text {bol }}\right)$.

Our $[\mathrm{Fe} / \mathrm{H}]$ values are systematically higher than those of the original analyses: the systematic difference is $0.12 \pm$ 0.01 dex $(\sigma=0.08,162$ stars $)$, as displayed also in Fig. 4 . This difference is mainly due to our use of K92 model atmospheres for both solar and stellar analysis. In fact, in previous analyses (e.g., both G8689 and SKPL) the solar Fe abundances were obtained using the HM model atmospheres, which is $\sim 150 \mathrm{~K}$ warmer than the BEGN models in the line formation region. We notice here that relative abundances (i.e. abundances obtained using model atmospheres from the same grid for both the Sun and the program stars) are almost insensitive to the grid adopted (differences are $<0.03 \mathrm{dex}$ ). In this respect, our analysis combines the advantages of both differential and absolute analyses, since our abundances are referred to the Sun, and we used a solar model extracted from the same grid of model atmospheres used for the program stars.

Table 6. Mean differences Fe I - Fe II in globular cluster giants

\begin{tabular}{lrcc}
\hline \hline & & & \\
Sample & Nr. Stars & $<$ Fe I - Fe II $>$ & $\sigma$ \\
& & & \\
\hline & & & \\
All & 147 & $0.00 \pm 0.01$ & 0.12 \\
CG96 & 10 & $-0.05 \pm 0.04$ & 0.11 \\
G8689 & 29 & $-0.10 \pm 0.03$ & 0.14 \\
SKPL & 82 & $+0.02 \pm 0.01$ & 0.08 \\
McW & 3 & $+0.13 \pm 0.04$ & 0.07 \\
M93 & 15 & $+0.11 \pm 0.04$ & 0.15 \\
NDC & 8 & $-0.01 \pm 0.02$ & 0.06 \\
& & & \\
\hline
\end{tabular}

Data concerning the Fe ionization equilibrium are shown in Table 6 which lists the mean differences between abundances derived from neutral and singly ionized lines of Fe. These values have been computed both for the total sample and for the different sub-samples studied. From this Table we conclude that there is an excellent agreement between abundances derived from Fe I and Fe II, with

$\overline{7}$ All stars that were in more than one data sets have been independently re-analyzed, and then their $[\mathrm{Fe} / \mathrm{H}]$ values averaged before computing the cluster's mean $[\mathrm{Fe} / \mathrm{H}]$. 
no trend with $T_{\text {eff }}$ or $[\mathrm{Fe} / \mathrm{H}]$. The lack of any trend over the whole range of temperature is very important, since in the past some analysis (e.g., Pilachowski et al. 1983) claimed that a discrepancy was present between these 2 iron abundances in stars cooler than $4300 \mathrm{~K}$. The implication was that in the very upper red giant branch the usual Local Thermodynamic Equilibrium (LTE) assumption had to be released or, at least, carefully verified by statistical equilibrium computations. Our results, however, strongly confirm the recent study of Clementini et al. (1995; see also the footnote below) that pointed out that departures from LTE cannot significatively affect abundance analyses for stars cooler than RR Lyrae variables.

For the SKPL sample it should be noted again that in their original papers both photometric gravities and $T_{\text {eff }}$ values were purposedly changed to obtain a match of the two $[\mathrm{Fe} / \mathrm{H}]$ abundances within 0.05 dex.

Table 7 shows the dependance of the derived abundances from uncertainties in the adopted atmospheric parameters; this is obtained by re-iterating the analysis while varying each time only one of the parameters. To show how these sensitivities change with overall metal abundance, we repeated this exercise for both a metal-rich (star 8406 in 47 Tuc) and a metal-poor giant (star A61 in NGC 6752).

Entities of variations are quoted in Table 7: these values are larger than errors likely present in the adopted atmospheric parameters. This will be shown in the following discussion, where we will try to provide reasonable evaluations for the uncertainties in the adopted atmospheric parameters. To this purpose, we compared expected scatters in Fe abundances within individual clusters and differences between abundances provided by neutral and singly ionized lines with observed values. Relevant data for this last parameter can be easily obtained from Table $6^{8}$. For the reasons above mentioned, we omit from the following discussion the value from the SKPL sample and we concentrate instead on the other mean differences, for which the standard deviation $\sigma=0.11 \div 0.15$ represents the random errors contribution, and the error of the mean (0.01 $\div 0.04)$ the contribution due to systematic errors.

\subsection{Systematic errors}

The relevance of systematic errors is always difficult to reliably assess. We do not think there are serious concerns related to the adopted $g f$ scale. On the other side, uncertainties due to the adopted model atmospheres may be large since various important aspects (like convection, molecular opacities, and horizontal inhomogeneities) are

\footnotetext{
8 Following the non-LTE analysis of Clementini et al. (1995), no significant departures from LTE are to be expected in RGB stars. The differences found in Fe abundances from Fe I and Fe II lines are thus likely to be interpreted as due to errors in the analysis and in the atmospheric parameters.
}

far from being adequately known. Large trends of Fe abundances with excitation have been obtained in the analysis of field metal-poor giants by Dalle Ore (1992), Dalle Ore et al. (1996), Gratton \& Sneden (1994), and Gratton et al. (1996), when using both BEGN and K92 model atmospheres. These trends suggest that currently available model atmospheres are not fully adequate for at least some metal-poor giants (see e.g. Castelli et al. 1996). While absolute abundances are quite sensitive to this source of errors, the comparison of relative abundances obtained with different model atmosphere grids (K92 and BEGN) suggests that our $[\mathrm{Fe} / \mathrm{H}]$ values are not heavily affected. However, our analysis should obviously be repeated once improved model atmospheres for metal-poor giants become available.

We need to concern less about possible errors in the adopted temperature scale (in our case, the CFP one). In fact, were the $T_{\text {eff }}$ scale largely in error, we would expect a rather large difference between average abundances provided by neutral and singly ionized Fe lines. The values listed in Col. 2 of Table 7 indicate that a systematic error of $100 \mathrm{~K}$ in the adopted $T_{\text {eff's }}$ would translate into a systematic difference of 0.2 dex between abundances of Fe I and Fe II. Since the observed difference ranges from 0.02 dex to 0.13 dex (depending on the considered sample), we conclude that the $T_{\text {eff }}$ scale cannot be systematically incorrect by more than $50 \mathrm{~K}$.

\subsection{Internal errors}

Internal errors may be determined from a comparison with the observed scatter in our abundance determinations (of individual lines and of individual stars in each cluster). We will consider only errors in the $E W \mathrm{~s}$ and in the adopted atmospheric parameters, while we regard internal errors in the adopted $g f_{\mathrm{s}}$ as negligible.

\subsubsection{Equivalent widths}

The scatter of abundances from individual (Fe I) lines is $0.13,0.11,0.15,0.15,0.14$ and $0.12 \mathrm{dex}$ for the CG96, SKPL, G8689, McW92, M 93 and NDC samples respectively. These values for the scatter can be ascribed to errors in the $E W \mathrm{~s}$ of a few $\mathrm{m} \AA$ (see Sect. 3), and yield mean internal errors of 0.03 and 0.06 dex for Fe I and Fe II respectively. These internal errors can be added quadratically and give a prediction of about 0.07 dex for the scatter in the differences between abundances derived from Fe I and Fe II lines. Since the observed scatter ranges from $\sigma=0.11$ to $\sigma=0.15$ (depending on the adopted sample), additional sources of errors are clearly present, probably related to the adopted values for the atmospheric parameters (see Table 7 and discussion below). 
Table 7. Dependence of the derived abundances on atmospheric parameters

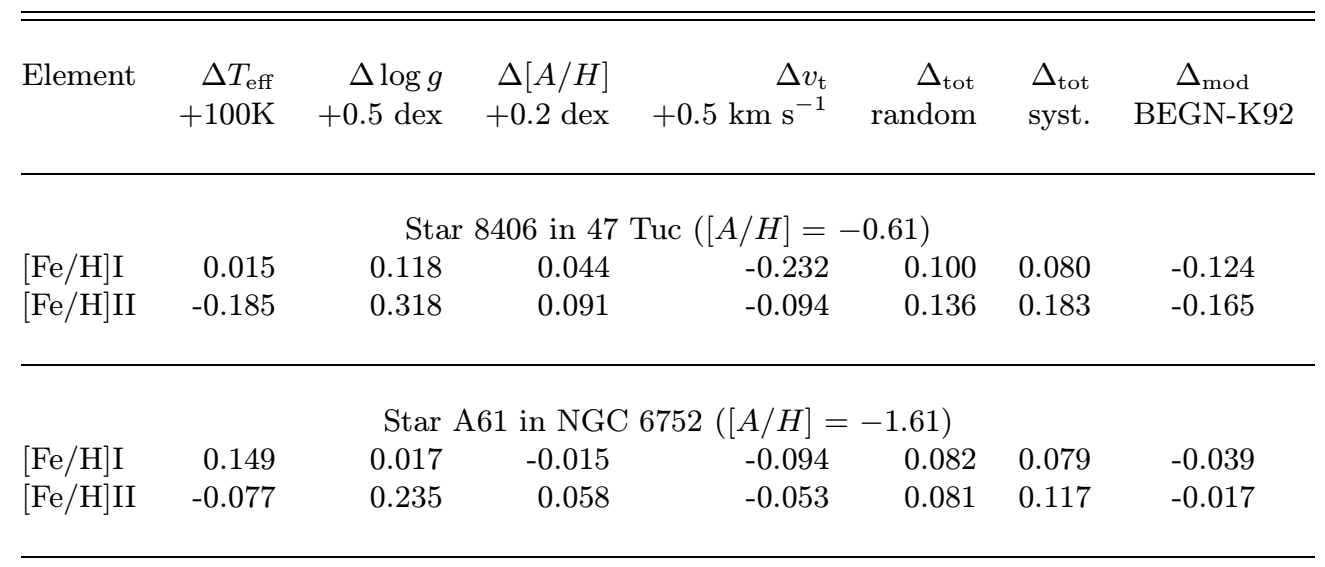

\subsection{Temperatures}

CFP $V-K$ colours have errors of $\sim 0.05 \mathrm{mag}$, which corresponds to $35-40 \mathrm{~K}$ using their calibration. This is the internal error of $T_{\text {eff's for stars within a cluster. When }}$ comparing stars in different clusters, the effects of errors in the interstellar reddening should also be considered. Comparing various estimates for the same cluster, we estimate an uncertainty of $0.02 \div 0.03 \mathrm{mag}$ in $E(B-V)$, and 2.7 times larger in $E(V-K)$. Hence, there is an additional systematic error of $\sim 0.05 \mathrm{mag}$ in the $(V-K)_{0}$ colour $(\sim 35-40 \mathrm{~K})$ systematic for all stars in a cluster (but random from cluster to cluster) due to errors in the reddening. If we add these two uncertainties quadratically, we estimate that the adopted $T_{\text {eff's }}$ have internal errors of $\sim 50 \mathrm{~K}$. The same figures approximately hold for the $B-V$ colour, which is a less accurate temperature indicator (see e.g., Gratton et al. 1996), but at the same time is measured with a precision better by a factor of 5 than the $V-K$ for bright globular cluster giants.

Table 7 suggests that most of the residual scatter in the differences between Fe I and Fe II abundances may be attributed to random errors in the adopted $T_{\text {eff }}$ values.

\subsection{Gravities}

The adopted gravities were deduced from the location of the stars in the CMD. Since they were not deduced from the ionization equilibrium, one could think that errors in $\log g$ and in $T_{\text {eff }}$ are not tied ${ }^{9}$. But, as matter of fact, temperature and gravity are not completely independent, since to derive $\log g$ from the position of the star in the

$9 \quad$ As a generic estimate, an error of $100 \mathrm{~K}$ in $T_{\text {eff }}$ translates into a 0.3 dex error in $\log g$, when deriving spectroscopically the gravities.
CMD we have to use the relationship $L=4 \pi R^{2} \sigma T_{\mathrm{eff}^{4}}$, i.e.:

$\log g / g_{\odot}=4 \log T_{\text {eff }} / T_{\odot}-\log L / L_{\odot}+\log M / M_{\odot}$

To estimate the order of magnitude of the errors affecting gravity, consider the following:

- the luminosity $\log L$ can be wrong either if the cluster distance moduli or the bolometric corrections $B C$ are wrong. The distance moduli were derived by CFP from a compilation of literature data; however uncertainties are not larger than $0.2 \mathrm{mag}$, i.e. 0.08 dex in $\log L$. The error in the $B C$ s depends on the model atmospheres, $T_{\text {eff }}$ 's, and metal abundances. It cannot be larger than $0.2-0.3 \mathrm{mag}$, though, else the luminosity at the tip of the RGB would disagree with that predicted by stellar evolution models. This contribution then translates into another 0.08 dex. If we sum these two contributions quadratically, we estimate that the total uncertainty in adopted luminosities is $\sim 0.11 \mathrm{dex}$ in $\log L$.

- the contribution of errors in the mass is very small, since this is fixed by the age of the globular clusters, and it cannot be wrong by more than $10 \%$. The uncertainty in $\log M$ may then be taken as 0.04 dex.

- as mentioned above, uncertainties in $T_{\text {eff's }}$, are of $\sim$ $50 \mathrm{~K}$, i.e. $\sim 0.005$ dex in $\log T_{\text {eff }}$.

From these considerations, we estimate that the adopted gravities have internal errors of $\sim 0.15$ dex.

In Col. 3 of Table 7 we investigate the effects of a variation of 0.5 dex in the surface gravity; on the basis of the previous discussion, the contribution from this column should be then divided by at least a factor of 3 . It is interesting to note that a larger error of $\Delta \log g=0.25$ would explain the whole residual $0.11 \mathrm{dex}$ in the random error. This is not the case, though, since there is surely a contribution from errors in $T_{\text {eff }}$ : this further confirms that 
$\Delta \log g=0.25$ is an overestimate, and the assumed value of 0.15 dex is reliable.

\subsection{Metallicities}

For each star analyzed we have also random errors in the estimate of $[A / H]$ due to errors in $T_{\text {eff }}$, in gravity (of little entity) and in the measured $E W$ s. This kind of errors can be evaluated from independent analyses of the same star. To this purpose, we can compare the results obtained for stars in the same cluster, since they are thought to share the same overall metallicity: the rms deviation from the mean will give an idea of the uncertainties due to random factors. The quadratic average is $0.06 \mathrm{dex}$ and so they contribute very little to the observed difference in the abundances from Fe I and Fe II (less than 0.025 dex, from Table 7).

\subsection{Microturbulent velocity}

The internal error in the $v_{\mathrm{t}}$ is usually estimated from the comparison of empirical and theoretical curve-of-growth; it is typically not larger than $0.2 \mathrm{~km} \mathrm{~s}^{-1}$ for the giants analyzed, since the microturbulent velocity is derived using Fe I lines both on the linear and saturation part of the curve-of-growth. As above, an independent test of the random errors comes from the comparison between the values obtained for the same star independently analyzed. We obtained $\Delta v_{\mathrm{t}}=0.17 \mathrm{~km} \mathrm{~s}^{-1}$ for the star C428 in CG96 and in the G8689 sample; it confirms that the microturbulent velocity has an error smaller than $0.2 \mathrm{~km} \mathrm{~s}^{-1}$.

\subsection{Discussion of errors}

To conclude, we have to consider two kinds of errors: first, the internal, random errors, that affect the comparison from star to star, and second, the systematic errors, that give an idea of the reliability of our metallicity scale, of the temperature scale adopted, etc. For the random errors, we have seen that reasonable estimates are $50 \mathrm{~K}$ in $T_{\text {eff }}, 0.15$ dex in $\log g, 0.06 \mathrm{dex}$ in $[A / H]$ and $0.2 \mathrm{~km} \mathrm{~s}^{-1}$ in $v_{t}$; these errors will affect the scatter of our data. As to systematic errors, we have only the indication given by the difference in the abundances from neutral and singly ionized Fe lines; from the previous discussion, we conclude that these errors are of the same order of magnitude of random ones.

Columns 6 and 7 of Table 7 list the uncertainties in the $[\mathrm{Fe} / \mathrm{H}]$ ratios derived from the quadratic sum of the contributions from random and systematic errors, respectively. We remark that the changes in the parameters used to construct these columns are not those indicated in the Table, but the more realistic estimates obtained from the above discussion. From Table 7 we can estimate that the total uncertainty in our Fe I abundances (from which we derive the clusters metallicity) is about 0.11 dex for the most metal-poor stars, increasing to about 0.13 dex for the most metal-rich stars.

\section{The metallicity scale of the globular clusters}

Mean values of the metallicities derived for the 21 clusters analyzed are listed in Table 8.

The internal uncertainty in $[\mathrm{Fe} / \mathrm{H}]$ abundances $\left(\sigma / N^{1 / 2}\right.$, where $N$ is the number of stars studied in each cluster) is very small: on average, $0.06 \mathrm{dex}$, which can be interpreted also as the mean precision of the cluster ranking on our new metallicity scale. For comparison, in the same Table, we also give the original $[\mathrm{Fe} / \mathrm{H}]$ ratios obtained in previous analyses. In the last column the metal abundances from the compilation of Zinn \& West (1984) are listed, superseded and integrated for a few clusters by the new measurements of Armandroff \& Zinn (1988); this scale will be indicated as a whole, hereinafter, as ZW.

\subsection{Comparison with the $Z W$ scale}

The 24 clusters of Table 8 can now be regarded as standard reference clusters to calibrate individual metal abundance indicators with metallicities directly derived from high-dispersion spectroscopic analysis. We feel confident that our list covers fairly well the whole range in metallicity of globular clusters, going from typical metal-rich clusters, as 47 Tuc, M 71 and NGC 6352, to the classical metal-poor templates (M 92, M 15, M 68). The sample of intermediate metallicity clusters is also very well represented among our calibrators. One of our main purposes is to revise and refine the calibration of the $\mathrm{ZW}$ ranking system, which covers almost all known globular clusters. The main advantage of the ZW scale is that their system is applicable even to the most distant objects, being based on the integrated parameter $Q_{39}$ and/or on low-dispersion spectroscopy of the infrared Ca II triplet. On the other side, any integrated index is not, by definition, a function of a single element in a globular cluster. In particular, the major contribution to line blanketing in the spectral range covered by the $Q_{39}$ index is due to the $\mathrm{H}$ and $\mathrm{K}$ lines of Ca II, with other significant fractions due to the $\lambda 3883$ $\mathrm{CN}$ band and some Fe blends. Hence, reliability of ZW metallicities ultimately rests on the coupling between $\mathrm{Ca}$, $\mathrm{C}, \mathrm{N}$, and Fe abundances. It is outside the purposes of this paper to proceed further on this point; we only wish to recall here that the strength of CN-bands is known to vary from star to star (the so-called $\mathrm{CN}$-signature), having a bimodal distribution in most (but not all!) clusters (see Kraft 1994 for a recent review).

Moreover (see e.g., Clementini et al. 1995, Sect. 5.1.1) the $[\mathrm{Ca} / \mathrm{Fe}]$ ratio does not scale with $\mathrm{Fe}$ on the whole range of metallicities, being lower in metal-rich than in metalpoor Population II stars. Furthermore, a serious caveat has been advanced on the claimed independence of the $Q_{39}$ index from the horizontal branch morphology (see 
Table 8. Mean metallicities for globular clusters compared to literature data

\begin{tabular}{|c|c|c|c|c|c|c|c|c|}
\hline NGC & Messier & Stars & Mean \pm rms & $\sigma$ & G8689 & SKPL & Others & ZW \\
\hline 104 & 47 Tuc & 5 & $-0.70 \pm 0.03$ & 0.07 & -0.82 & & & -0.71 \\
\hline 288 & & 2 & $-1.07 \pm 0.03$ & 0.04 & -1.31 & & & -1.40 \\
\hline 362 & & 1 & -1.15 & & -1.18 & & & -1.27 \\
\hline 1904 & M 79 & 2 & $-1.37 \pm 0.00$ & 0.01 & -1.42 & & & -1.68 \\
\hline 2298 & & 3 & $-1.74 \pm 0.04$ & 0.06 & & & $-1.91^{a}$ & -1.85 \\
\hline 3201 & & 3 & $-1.23 \pm 0.05$ & 0.09 & -1.34 & & & -1.61 \\
\hline 4590 & M 68 & 3 & $-1.99 \pm 0.06$ & 0.10 & -1.92 & & $-2.17^{b}$ & -2.09 \\
\hline 4833 & & 3 & $-1.58 \pm 0.01$ & 0.01 & -1.74 & & $-1.71^{b}$ & -1.86 \\
\hline 5272 & M 3 & 10 & $-1.34 \pm 0.02$ & 0.06 & & -1.46 & & -1.66 \\
\hline 5897 & & 2 & $-1.59 \pm 0.03$ & 0.05 & -1.84 & & & -1.68 \\
\hline 5904 & M 5 & 16 & $-1.11 \pm 0.03$ & 0.11 & -1.42 & -1.17 & & -1.40 \\
\hline 6121 & M 4 & 3 & $-1.19 \pm 0.03$ & 0.06 & -1.32 & & & -1.33 \\
\hline 6144 & & 1 & -1.49 & & & & $-1.59^{b}$ & -1.75 \\
\hline 6205 & M 13 & 23 & $-1.39 \pm 0.01$ & 0.07 & & -1.49 & & -1.65 \\
\hline 6254 & M 10 & 15 & $-1.41 \pm 0.02$ & 0.10 & -1.42 & -1.52 & & -1.60 \\
\hline 6341 & M 92 & 9 & $-2.16 \pm 0.02$ & 0.05 & & -2.25 & & -2.24 \\
\hline 6352 & & 3 & $-0.64 \pm 0.06$ & 0.11 & -0.79 & & & -0.51 \\
\hline 6362 & & 2 & $-0.96 \pm 0.00$ & 0.01 & -1.04 & & & -1.08 \\
\hline 6397 & & 10 & $-1.82 \pm 0.04$ & 0.10 & -1.88 & & & -1.91 \\
\hline 6656 & & 3 & $-1.48 \pm 0.03$ & 0.06 & -1.56 & & & -1.75 \\
\hline 6752 & & 12 & $-1.42 \pm 0.02$ & 0.08 & -1.53 & & & -1.54 \\
\hline 6838 & M 71 & 13 & $-0.70 \pm 0.03$ & 0.09 & -0.81 & -0.79 & & -0.58 \\
\hline 7078 & M 15 & 4 & $-2.12 \pm 0.01$ & 0.01 & & -2.30 & $-2.23^{b}$ & -2.17 \\
\hline 7099 & M 30 & 2 & $-1.91 \pm 0.00$ & 0.00 & & & $-2.11^{b}$ & -2.13 \\
\hline
\end{tabular}

References: $\mathrm{a}=\mathrm{McW} 92, \mathrm{~b}=\mathrm{M} 93$.

e.g., Smith 1984). To overcome this kind of problems, the most straightforward way to correct the ZW scale consists in working directly on the final metallicities, since the original compilation of Zinn \& West (1984) was obtained averaging a number of $[\mathrm{Fe} / \mathrm{H}]$ values derived from different indicators (e.g., $\left.(B-V)_{0, g}, \Delta V_{1.4}, \Delta S\right)$ and calibrated against $Q_{39}$.

In Fig. 5 we then compare our high-dispersion $[\mathrm{Fe} / \mathrm{H}]$ values with the $\mathrm{ZW}$ values for the 24 calibrating clusters. The error bars $\left(\begin{array}{ll}1 & \sigma\end{array}\right)$ are from Zinn \& West (1984: Table 5) and from our Table 8. As it is evident from this figure, the ZW scale is far from linear, deviating both in the low and in the high metallicity regimes, when compared with $[\mathrm{Fe} / \mathrm{H}]$ from our direct analysis. In the metal-rich region $([\mathrm{Fe} / \mathrm{H}]>-1)$ ZW's metallicities are on average 0.08 dex too high for the 3 clusters 47 Tuc, M 71 and NGC 6352, with the last two objects being responsible for most of the discrepancy $(0.12$ and 0.13 dex, respectively). For $-1 \leq[\mathrm{Fe} / \mathrm{H}] \leq-1.9$ the $[\mathrm{Fe} / \mathrm{H}]$ values of $\mathrm{ZW}$ are definitively too low by a mean value of 0.23 dex ( $\sigma=0.09$ dex, 16 clusters). Finally, in the very low-metallicity tail, ZW's values are on average $0.11 \mathrm{dex}$ higher than ours ( $\sigma=0.06$ dex, 6 clusters).

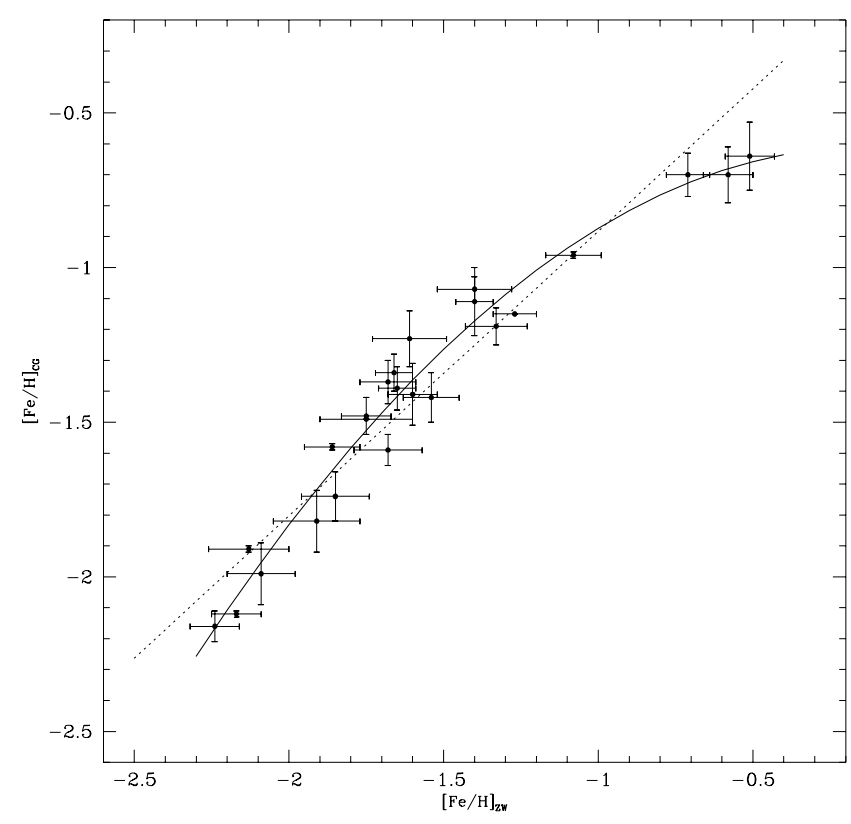

Fig. 5. Mean metallicities for the 24 clusters from the present work compared with metallicities on the Zinn \& West scale (1984) 
The non-linear behaviour has been confirmed by a $t$-test on the significance of the quadratic term in the relation between $\mathrm{ZW}$ and ours $[\mathrm{Fe} / \mathrm{H}]$ 's. To bring ZW's $[\mathrm{Fe} / \mathrm{H}]$ ratios on a metallicity scale fully based only on high dispersion spectroscopy (HDS) we then derived a correction given by a quadratic relation. This procedure automatically takes into account also the different zero point between the two scales, since the ZW scale was ultimately based on the Cohen (1983) scale, which, as other past analysis, adopts the traditional old solar Fe abundance $\log \epsilon=7.67$. The resulting function we derive for this correction is:

$$
\begin{array}{cc}
{[\mathrm{Fe} / \mathrm{H}]_{\mathrm{CG}}} & =-0.618( \pm 0.083) \\
& -0.097( \pm 0.189)[\mathrm{Fe} / \mathrm{H}]_{\mathrm{ZW}} \\
-0.352( \pm 0.067)[\mathrm{Fe} / \mathrm{H}]_{\mathrm{ZW}}{ }^{2}
\end{array}
$$

with the correlation coefficient $r=0.982$ and $\sigma=0.08$ for 24 clusters. This relationship is highly significant, from a statistical point of view, and can be applied to ZW metallicities in the range $-2.24<[\mathrm{Fe} / \mathrm{H}]_{\mathrm{ZW}}<-0.51$, defined by the lowest and highest values of $[\mathrm{Fe} / \mathrm{H}]_{\mathrm{ZW}}$ among the clusters used for the calibration. The quadratic regression line is shown as a heavy line in Fig. 5; overimposed in the same figure is also the result of a linear fit, which takes into account the errors. As one can see, even considering $3 \sigma$ error bars, it it very difficult to represent the data on a linear scale, in particular at the lower metallicity edge.

Once the correction is applied the non-linearity of the ZW scale obviously disappears. However, a certain amount of scatter is still present in the intermediate-metallicity regime; we believe that it could be attributed to a residual effect, not well removed by our calibration, of the second parameter. This last is in fact likely to affect ZW's metallicities more severely in this regime, in which the integrated colours of clusters of different HB morphological type can be sensibly misinterpreted in terms of $[\mathrm{Fe} / \mathrm{H}]$.

The next logical step would be now to calibrate other empirical metallicity indicators, i.e. repeat the original work of Zinn \& West (1984) but using now our direct $[\mathrm{Fe} / \mathrm{H}]$ values from HDS as a calibrating sequence. The most interesting and accessible parameters are the photometric ones (e.g., $(B-V)_{0, g}, \Delta V_{1.4}$, etc.): they are widely used since it is easy enough to measure them from the recent and accurate CCD-based colour-magnitude diagrams (CMD). However, it would be preferable to have a dataset of homogeneity and accuracy comparable with the precision of our metallicities, instead of relying on compilation from different sources. Since such an effort is presently in progress on a set of CMDs analyzed in a self-consistent way, we postpone to a forthcoming paper this kind of calibration. However, an immediate and meaningful comparison can be made with the metallicity scale for globular clusters derived from RR Lyraes, since we can compare results obtained for two different stellar populations, RGB stars and $\mathrm{HB}$ stars, independently checking the validity of both scales.

\subsection{Comparison with the metallicity scale of RR Lyrae stars}

The most recent calibration of $[\mathrm{Fe} / \mathrm{H}]$ in terms of the Preston's (1959) index $\Delta S$ is the one defined by Clementini et al. (1995), who found

$[\mathrm{Fe} / \mathrm{H}]=-0.194( \pm 0.011) \Delta S-0.08( \pm 0.18)$

This relation was derived using RR Lyraes both in the field and in globular clusters. However, while metallicity values for field RR Lyraes were directly derived from highresolution spectra or from the re-analysis of literature data (for a total of $28 \mathrm{RR}$ Lyraes), cluster metallicities were taken at face value from the literature, even if a zero point was admittedly noted while using data from different samples. We have many clusters in common with the study of Clementini et al. (1995) and have then derived again the $[\mathrm{Fe} / \mathrm{H}]$ vs $\Delta S$ relation. Figure 6 shows the result of our re-analysis.

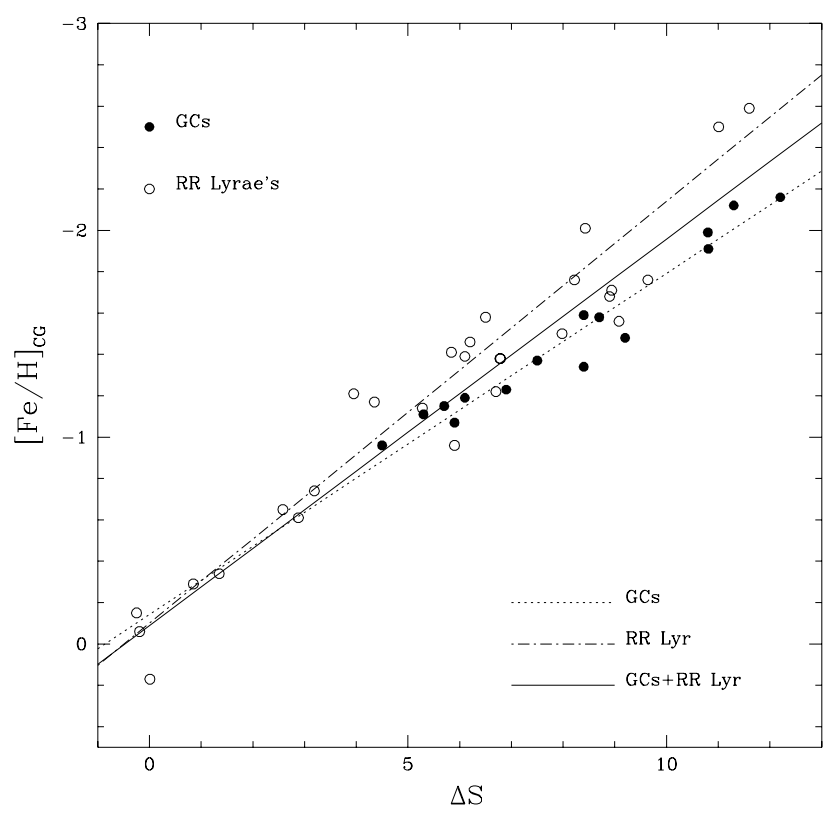

Fig. 6. Calibration of the $\Delta S$ index with our new analysis and with the data of field RR Lyrae variables from Clementini et al. (1995)

We obtained $\Delta S$ values for 15 of our calibrating clusters from the metallicities of Costar \& Smith (1988), inverting the Butler's (1975) relation they used. Our values are not completely identical to those used by Clementini et al. (1995); the main differences are that a) we excluded 47 Tuc, since its value for $\Delta S$ is based on a single star, possibly not member of the cluster (Tucholke 1992) and 
b) for NGC 288 we assumed a value of $\Delta S=5.9$, since the mean value 7.2 cited by Costar \& Smith was obtained including spectra taken at phases near maximum light.

Regression lines were then obtained by least-squares fits (we averaged values obtained exchanging the independent and dependent variables):

- If we consider only the 15 GCs we obtain:

$[\mathrm{Fe} / \mathrm{H}]=-0.165( \pm 0.019) \Delta S-0.142( \pm 0.033)$

with a $\sigma=0.130$, and $r=0.947$ (dotted line in Fig. 6).

- It we consider both the 15 GCs and the 28 field RR Lyraes we get:

$[\mathrm{Fe} / \mathrm{H}]=-0.187( \pm 0.011) \Delta S-0.088( \pm 0.041)$

with a $\sigma=0.269$, and $r=0.954$ (solid line in Fig. 6).

Also shown in Fig. 6 is the calibration obtained by Clementini et al. (1995), using only 28 field variables (their Eq. (6)): $[\mathrm{Fe} / \mathrm{H}]=-0.204( \pm 0.012) \Delta S-0.102( \pm 0.036)$, $\sigma=0.190$.

The first striking evidence both from Fig. 6 and the above equations is that the sequence of the globular cluster points seems to be much better defined, with a smaller scatter than the distribution of field RR Lyraes. The scatter in Eq. (6) of Clementini et al. is 0.19 dex, to be compared with the value of 0.13 dex obtained using only the new values for the clusters. We stress the fact that both the solar Fe abundance and the source for the oscillator strengths are in common between the present analysis and that of Clementini et al. (1995); moreover, the procedure followed in the abundance analysis is virtually the same. This may be evidence in favour of a larger intrinsic scatter in field than in cluster variables, or it may just reflect a smaller error in the values of $\Delta S$ for cluster RR Lyraes. However the last explanation seems a little unpalatable, since determinations of $\Delta S$ values are usually more accurate for nearby field stars.

The second feature shown in Fig. 6 is a rather clear separation between the relations for cluster and field RR Lyraes in the low metallicity region; this is the likely explanation for the increase in the scatter when the calibration $[\mathrm{Fe} / \mathrm{H}]-\Delta S$ is made using both cluster and field variables. The same behaviour was evident also in Fig. 14c of Clementini et al. (1995), but here it is even clearer, given the high degree of homogeneity in our data. Why this is so, we are not sure, apart from a suggestion of non-linearity in the $\Delta S-[\mathrm{Fe} / \mathrm{H}]$ relation theoretically predicted (Manduca 1981) and discussed in Clementini et al. (1995). Apart from this, there seems to be a good agreement between both scales; if we use our new calibration (Eq. 12) to derive $[\mathrm{Fe} / \mathrm{H}]$ ratios, the differences $[\mathrm{Fe} / \mathrm{H}]_{\mathrm{CG}}$ $-[\mathrm{Fe} / \mathrm{H}]_{\Delta S}$ are on average $0.12 \pm 0.03(\sigma=0.10$, for 16 clusters).

\section{Discussion and conclusions}

We think that the effort to get more reliable and accurate metallicities is truly worthwhile: a new, homogeneous scale of $[\mathrm{Fe} / \mathrm{H}]$ for globular clusters is really needed, since up to now the numerous but still heterogeneous estimates from HDS have been systematically ignored in many problems of stellar evolution.

As an illustrative example we will explore the effects of our new $[\mathrm{Fe} / \mathrm{H}]$ values on the long-standing problem of the Oosterhoff (1944) effect among globular clusters, which belong to 2 groups on the basis of the mean period of their type $a b$ RR Lyraes; this division reflects a separation in metallicity of the clusters (Arp 1955). The statement of this problem, its history and references are fully addressed in a recent paper by Sandage (1993a). Briefly, the concept is to use the pulsation equation for RR Lyrae stars $(P=$ $f\left(T_{\text {eff }}, L, M\right)$, where $P$ is the fundamental period of the pulsation, $L$ the luminosity and $M$ the mass of the star) to derive a calibration of the absolute luminosity of RR Lyraes in terms of the metal content, $[\mathrm{Fe} / \mathrm{H}]$, in turn a cornerstone to ultimately get the ages of globular clusters. This is possible if the variations of the parameters involved $\left(P, T_{\text {eff }}, L, M\right)$ with $[\mathrm{Fe} / \mathrm{H}]$ are known.

The critical and more debated point is to determine how $\Delta \log P$, the shift existing in the $\log P-\log T_{\text {eff }}$ plane between the distribution of variables in OoI and OoII clusters, varies with the metallicity; the slope, in particular, is still much controversial. While Sandage (1993a) finds a value of about 0.12 , theoretical models seem to predict a much lower value, around 0.05 (in the sense of longer periods for metal-poorer clusters). Our approach is based both on our new metallicities and new $T_{\text {eff }}$ 's for variables in M 3 (OoI) and M 15 (OoII), i.e. the template-pair for the Oosterhoff effect. ${ }^{10}$

(a) From the present work we adopt $[\mathrm{Fe} / \mathrm{H}]=-1.34$ for $\mathrm{M} 3$ and $[\mathrm{Fe} / \mathrm{H}]=-2.12$ for $\mathrm{M} 15$. These values compare well to those derived by Sneden et al. (1991) and Kraft et al. $(1992,[\mathrm{Fe} / \mathrm{H}]=-1.48$ and -2.30 for $\mathrm{M} 3$ and $\mathrm{M} 15$ respectively), allowing for their use of the old Bell et al. (1976) model atmospheres and of a different set of $g f$.

If one compares these new values with the classical ZW ones ( -1.66 for M 3 and -2.15 for M 15), it it immediately evident that whatever the period shift between $M 3$ and $M$ 15 may be, it has to be "diluted" on a larger range of metallicity than before. This in turn affects the slope of the relationship $\log P-[\mathrm{Fe} / \mathrm{H}]$.

b) We adopt here the new temperature scale derived by Gratton et al. (1996). Briefly, they first derived empirical colour- $T_{\text {eff }}$ relations for population I stars, based on $T_{\text {eff }}$ 's from the Infrared Flux Method (IRFM, Blackwell \& Lynas Gray 1994; Bell \& Gustafsson 1989) and interferometric diameters (Di Benedetto \& Rabbia 1987); stars of luminosity class III and V were considered separately. $T_{\text {eff }}$ 's for stars of different $\log g$ and $[\mathrm{Fe} / \mathrm{H}]$ are then

\footnotetext{
10 Our conclusions would not change had we used M 68 $([\mathrm{Fe} / \mathrm{H}]=-1.99$ on our scale $)$ instead of M 15 as OoII template. While M 68 has a small and well determined reddening $(E(B-V)=0.03), \mathrm{M} 15$ has a better populated instability strip.
} 
Table 9. Adopted atmospheric parameters and results of abundance analysis for globular cluster giants

\begin{tabular}{|c|c|c|c|c|c|c|c|c|c|c|c|c|c|}
\hline NGC & Star & Source & Teff & $\log g$ & {$[A / H]$} & $\mathrm{Vt}$ & $\mathrm{r}$ & $\mathrm{FeI}$ & $\sigma(\mathrm{FeI})$ & $\mathrm{r}$ & FeII & $\sigma(\mathrm{FeII})$ & {$[\mathrm{Fe} / \mathrm{H}]$} \\
\hline 104 & 1407 & G8689 & 4500 & 2.00 & -0.73 & 1.53 & 18 & 6.79 & 0.12 & 1 & 6.84 & & -0.73 \\
\hline 104 & 8416 & G8689 & 4425 & 1.80 & -0.82 & 1.79 & 12 & 6.70 & 0.17 & 2 & 6.66 & 0.28 & -0.82 \\
\hline 104 & 8406 & CG95 & 4040 & 1.10 & -0.61 & 1.76 & 35 & 6.92 & 0.18 & 3 & 6.70 & 0.10 & -0.60 \\
\hline 104 & 5422 & CG95 & 4090 & 1.20 & -0.70 & 1.62 & 35 & 6.83 & 0.15 & 2 & 6.90 & 0.01 & -0.69 \\
\hline 104 & 5529 & CG95 & 3850 & 0.70 & -0.66 & 1.59 & 6 & 6.86 & 0.16 & 2 & 6.88 & 0.26 & -0.66 \\
\hline 288 & $\begin{array}{l}245 \\
231\end{array}$ & G8689 & 4400 & 1.20 & -1.10 & 1.55 & 21 & 6.41 & 0.15 & & & & -1.11 \\
\hline $\begin{array}{l}288 \\
362\end{array}$ & $\begin{array}{r}231 \\
\mathrm{I}-23\end{array}$ & $\begin{array}{r}\text { G8689 } \\
\text { G8689 }\end{array}$ & $\begin{array}{l}4475 \\
4335\end{array}$ & $\begin{array}{l}1.30 \\
1.10\end{array}$ & $\begin{array}{l}-1.03 \\
-1.15\end{array}$ & $\begin{array}{l}1.42 \\
1.55\end{array}$ & $\begin{array}{l}19 \\
23\end{array}$ & 6.49 & 0.19 & & & & -1.03 \\
\hline $\begin{array}{r}362 \\
1904\end{array}$ & $\begin{array}{r}-23 \\
223\end{array}$ & $\begin{array}{r}\text { G86689 } \\
\text { G8689 }\end{array}$ & $\begin{array}{l}4335 \\
4250\end{array}$ & $\begin{array}{l}1.10 \\
0.75\end{array}$ & $\begin{array}{l}-1.15 \\
-1.36\end{array}$ & $\begin{array}{l}1.55 \\
1.69\end{array}$ & $\begin{array}{l}23 \\
20\end{array}$ & $\begin{array}{l}6.37 \\
6.16\end{array}$ & $\begin{array}{l}0.16 \\
0.19\end{array}$ & 7 & 6.16 & 0.21 & $\begin{array}{l}-1.15 \\
-1.36\end{array}$ \\
\hline 1904 & 153 & G8689 & 4270 & 0.75 & $\begin{array}{l}-1.30 \\
-1.38\end{array}$ & $\begin{array}{l}1.05 \\
2.23\end{array}$ & 17 & 6.15 & $\begin{array}{l}0.19 \\
0.15\end{array}$ & & & 0.21 & $\begin{array}{l}-1.36 \\
-1.37\end{array}$ \\
\hline 2298 & 7 & MCW92 & 4351 & 0.80 & -1.64 & 1.49 & 24 & 5.89 & 0.17 & 2 & 5.77 & 0.14 & $\begin{array}{l}-1.57 \\
-1.63\end{array}$ \\
\hline & & & & & & & 23 & 5.74 & 0.13 & 2 & 5.51 & 012 & $\begin{array}{l}1.78 \\
-1.78\end{array}$ \\
\hline 2298 & 9 & MCW92 & 4348 & 1.00 & -1.81 & 1.51 & 23 & 5.70 & 0.15 & ${ }_{2}^{2}$ & 5.65 & 0.07 & $\begin{array}{l}-1.18 \\
-1.82\end{array}$ \\
\hline 3201 & 3522 & G8689 & 4450 & 1.20 & -1.35 & 1.76 & 17 & 6.17 & 0.10 & 4 & 6.22 & 0.02 & -1.35 \\
\hline 3201 & 1501 & G8689 & 4445 & 1.20 & -1.15 & 1.58 & 18 & 6.36 & 0.15 & 5 & 6.34 & 0.21 & -1.16 \\
\hline 3201 & 1410 & G8689 & 4480 & 1.20 & -1.19 & 0.98 & 11 & 6.33 & 0.19 & & 0.07 & & -1.19 \\
\hline 4590 & 260 & M93 & 4329 & 0.70 & -2.09 & 2.16 & 27 & 5.42 & 0.11 & 3 & 5.23 & 0.15 & -2.10 \\
\hline 4590 & 53 & M93 & 4400 & 1.00 & -2.09 & 1.79 & 26 & 5.42 & 0.13 & 3 & 5.14 & 0.21 & -2.10 \\
\hline 4590 & I-260 & G8689 & 4329 & 0.75 & -1.63 & 0.82 & 11 & 5.90 & 0.13 & 2 & 5.88 & 0.01 & -1.63 \\
\hline 4590 & $\mathrm{I}-256$ & G8689 & & 0.80 & -2.01 & 1.66 & 7 & 5.51 & 0.05 & 3 & 5.70 & 0.06 & -2.01 \\
\hline 4833 & 13 & M93 & 4500 & 1.30 & -1.59 & 1.78 & 23 & 5.93 & 0.12 & 1 & 5.74 & & -1.59 \\
\hline 4833 & B172 & G8689 & 4323 & 0.80 & -1.56 & 1.72 & 18 & 5.96 & 0.16 & 5 & 6.08 & 0.13 & -1.56 \\
\hline 4833 & MA-1 & G8689 & 4273 & 0.75 & -1.59 & 1.90 & 21 & 5.94 & 0.14 & 6 & 6.10 & 0.19 & -1.58 \\
\hline 5272 & II- 46 & SKPL & 4000 & 0.60 & -1.41 & 2.02 & 15 & 6.12 & 0.06 & 4 & 6.14 & 0.14 & -1.40 \\
\hline 5272 & 297 & SKPL & 4070 & 0.70 & -1.39 & 1.93 & 12 & 6.14 & 0.16 & 5 & 6.14 & 0.10 & -1.38 \\
\hline 5272 & & SKPL & 4000 & 0.40 & -1.32 & 2.09 & 17 & 6.20 & 0.11 & 5 & 6.23 & 0.07 & -1.32 \\
\hline 5272 & MB-3 & SKPL & 3900 & 0.20 & -1.30 & 1.76 & 14 & 6.22 & 0.15 & 4 & 6.38 & 0.11 & -1.30 \\
\hline 5272 & MB-4 & SKPL & 3925 & 0.30 & -1.23 & 1.93 & 18 & 6.30 & 0.20 & 4 & 6.39 & 0.21 & -1.22 \\
\hline 5272 & 1000 & SKPL & 4175 & 0.45 & -1.38 & 1.98 & 15 & 6.14 & 0.11 & 4 & 6.20 & 0.14 & -1.38 \\
\hline 5272 & 1127 & SKPL & 4225 & 0.90 & -1.34 & 1.80 & 17 & 6.19 & 0.11 & 4 & 6.14 & 0.10 & -1.33 \\
\hline 5272 & 1397 & SKPL & 3950 & 0.40 & -1.32 & 2.13 & 17 & 6.20 & 0.12 & 4 & 6.20 & 0.12 & -1.32 \\
\hline 5272 & III-28 & SKPL & 4160 & 0.75 & -1.45 & 1.69 & 14 & 6.07 & 0.15 & 5 & 6.10 & 0.18 & -1.45 \\
\hline $\begin{array}{l}5272 \\
5897\end{array}$ & $\begin{array}{c}\text { MB-1 } \\
9\end{array}$ & $\begin{array}{l}\text { SKPL } \\
\text { G8689 }\end{array}$ & $\begin{array}{l}3825 \\
4175\end{array}$ & 0.00 & $\begin{array}{l}-1.34 \\
-1.64\end{array}$ & $\begin{array}{l}2.34 \\
1.86\end{array}$ & 8 & $\begin{array}{l}6.19 \\
588\end{array}$ & 0.18 & 2 & 6.24 & 0.08 & -1.33 \\
\hline $\begin{array}{l}5897 \\
5897\end{array}$ & $\begin{array}{r}9 \\
160\end{array}$ & $\begin{array}{l}\text { G88689 } \\
\text { G88899 }\end{array}$ & $\begin{array}{l}4175 \\
4300\end{array}$ & $\begin{array}{l}0.75 \\
0.80\end{array}$ & $\begin{array}{l}-1.64 \\
-1.55\end{array}$ & $\begin{array}{l}1.86 \\
1.67\end{array}$ & $\begin{array}{l}12 \\
17\end{array}$ & $\begin{array}{l}5.88 \\
5.98\end{array}$ & $\begin{array}{l}0.13 \\
0.13\end{array}$ & $\begin{array}{l}2 \\
3\end{array}$ & $\begin{array}{l}6.08 \\
6.17\end{array}$ & $\begin{array}{l}0.03 \\
0.17\end{array}$ & $\begin{array}{l}-1.64 \\
1.55\end{array}$ \\
\hline $\begin{array}{l}5897 \\
5904\end{array}$ & $\begin{array}{r}160 \\
\text { IV-47 }\end{array}$ & $\begin{array}{l}\text { G8689 } \\
\text { SKPL }\end{array}$ & $\begin{array}{l}4300 \\
4110\end{array}$ & $\begin{array}{l}0.80 \\
0.90\end{array}$ & $\begin{array}{l}-1.55 \\
-1.04\end{array}$ & $\begin{array}{l}1.67 \\
1.75\end{array}$ & $\begin{array}{l}17 \\
18\end{array}$ & $\begin{array}{l}5.98 \\
6.48\end{array}$ & $\begin{array}{l}0.13 \\
0.11\end{array}$ & $\begin{array}{l}3 \\
5\end{array}$ & $\begin{array}{l}6.17 \\
6.48\end{array}$ & $\begin{array}{l}0.17 \\
0.11\end{array}$ & $\begin{array}{l}-1.55 \\
-1.04\end{array}$ \\
\hline 5904 & II -85 & SKPL & 4050 & 0.90 & $\begin{array}{l}-1.00 \\
-1.00\end{array}$ & $\begin{array}{l}1.75 \\
1.75\end{array}$ & $\begin{array}{l}18 \\
19\end{array}$ & $\begin{array}{l}.48 \\
6.52\end{array}$ & 0.11 & $\begin{array}{l}5 \\
5\end{array}$ & $\begin{array}{l}0.48 \\
6.54\end{array}$ & $\begin{array}{l}0.11 \\
0.07\end{array}$ & $\begin{array}{l}-1.04 \\
-1.00\end{array}$ \\
\hline 5904 & III- 122 & SKPL & 4050 & 0.70 & -1.09 & 2.05 & 16 & 6.43 & 0.12 & 4 & 6.46 & 0.15 & -109 \\
\hline 5904 & III-3 & SKPL & 4070 & 0.75 & -1.15 & 1.99 & 18 & 6.37 & 0.10 & 4 & 6.46 & 0.12 & -1.15 \\
\hline 5904 & IIII-96 & SKPL & 4300 & 1.30 & -1.07 & 1.61 & 18 & 6.46 & 0.09 & 5 & 6.41 & 0.07 & -1.06 \\
\hline 5904 & IV-72 & SKPL & 4300 & 1.20 & -1.01 & $\begin{array}{l}1.59 \\
1.59\end{array}$ & 16 & 6.51 & 0.10 & 5 & 6.45 & 0.06 & -1.01 \\
\hline 5904 & II-9 & SKPL & 4300 & 0.80 & - & 1.78 & 13 & 6.54 & 0.16 & 3 & 6.64 & .08 & -0.98 \\
\hline 5904 & $\mathrm{I}-68$ & SKPL & 4130 & 0.90 & -1.09 & 2.01 & 17 & 6.44 & 0.15 & 4 & 6.43 & 0.15 & -1.09 \\
\hline 5904 & III-78 & SKPL & 4200 & 1.00 & -1.03 & 1.86 & 18 & 6.49 & 0.16 & 6 & 6.46 & 0.09 & -1.03 \\
\hline 5904 & III-36 & SKPL & 4250 & 1.10 & -1.06 & 1.61 & 18 & 6.46 & 11 & 5 & 6.40 & 0.08 & -1.06 \\
\hline 5904 & I-4 & G8689 & 4385 & 1.30 & -1.41 & 1.97 & 21 & 6.11 & 0.10 & 3 & 6.26 & 0.04 & -1.41 \\
\hline $\begin{array}{l}5904 \\
5904\end{array}$ & $\begin{array}{l}\mathrm{I}-25 \\
\mathrm{I}-61\end{array}$ & $\begin{array}{r}\text { G8689 } \\
\text { G8689 }\end{array}$ & 4435 & 1.40 & -1.14 & 1.68 & 13 & 6.37 & 0.16 & 4 & 645 & 007 & -1.15 \\
\hline $\begin{array}{l}5904 \\
5904\end{array}$ & $\begin{array}{r}\text { I-61 } \\
\text { IV-59 }\end{array}$ & $\begin{array}{l}\text { G8689 } \\
\text { SKPL }\end{array}$ & $\begin{array}{l}4380 \\
4320\end{array}$ & $\begin{array}{l}1.30 \\
1.10\end{array}$ & $\begin{array}{l}-1.31 \\
-1.12\end{array}$ & $\begin{array}{l}1.92 \\
2.19\end{array}$ & $\begin{array}{l}23 \\
15\end{array}$ & $\begin{array}{l}6.21 \\
6.39\end{array}$ & $\begin{array}{l}0.12 \\
0.12\end{array}$ & $\begin{array}{l}4 \\
3\end{array}$ & $\begin{array}{l}6.45 \\
6.36\end{array}$ & $\begin{array}{l}0.07 \\
0.06\end{array}$ & $\begin{array}{l}-1.31 \\
\end{array}$ \\
\hline 5904 & I-71 & $\begin{array}{l}\text { SKPL } \\
\text { SKP }\end{array}$ & $\begin{array}{l}4320 \\
4390\end{array}$ & $\begin{array}{l}1.10 \\
1.30\end{array}$ & $\begin{array}{l}-1.12 \\
-1.04\end{array}$ & $\begin{array}{l}2.19 \\
1.55\end{array}$ & $\begin{array}{l}15 \\
17\end{array}$ & $\begin{array}{l}.39 \\
6.48\end{array}$ & $\begin{array}{l}0.12 \\
0.07\end{array}$ & $\begin{array}{l}3 \\
5\end{array}$ & $\begin{array}{l}6.36 \\
6.44\end{array}$ & $\begin{array}{l}0.06 \\
0.11\end{array}$ & $\begin{array}{l}-1.13 \\
-1.05\end{array}$ \\
\hline 5904 & III-50 & SKPL & 4400 & 1.00 & -1.22 & 1.96 & 18 & 6.30 & 0.11 & 5 & 6.30 & 0.11 & -1.22 \\
\hline 612 & 2626 & G8689 & 4295 & 1. & -1.27 & 1.5 & 20 & 6.26 & 0.16 & 2 & 6.65 & 0.01 & -1.26 \\
\hline 6121 & 2608 & G8689 & 4285 & 1.5 & -1.14 & 1.3 & 21 & 6.38 & 0. & 2 & 6.66 & 0.08 & -1.14 \\
\hline 6121 & 1605 & G8689 & & 1. & -1 & & 22 & 6. & & 1 & 6.40 & & -1.16 \\
\hline 61 & 152 & M93 & 4600 & 1.50 & -1.49 & 1.53 & 20 & 6.03 & 0.13 & 2 & 6.04 & 0.12 & -1.49 \\
\hline 6205 & 262 & SKPL & 4180 & 0.80 & -1.40 & 1.90 & 18 & 6.13 & 0.10 & 5 & 6.12 & 0.11 & -1.39 \\
\hline 6205 & III- 56 & SKPL & 4100 & 0.65 & - & 2.01 & 17 & 6.11 & 0.10 & 5 & 6.16 & 10 & -1.41 \\
\hline 6205 & $\therefore$ & SK & 4180 & 0.8 & -1 & 1. & 17 & 6.14 & & 5 & 6.08 & 09 & -1.39 \\
\hline 6205 & 598 & SKPL & 3900 & 0.00 & -1.45 & 2.11 & 17 & 6.08 & 0.15 & 4 & 6.17 & 0.12 & -1.44 \\
\hline 6205 & 261 & SKPL & 4230 & 0.85 & -1.36 & 2.01 & 17 & 6.16 & 9 & 4 & 6.03 & .05 & -1.36 \\
\hline 6205 & $\begin{array}{r}835 \\
\end{array}$ & $\begin{array}{l}\text { SKPL } \\
\text { SKPI }\end{array}$ & 4090 & 0.70 & $\begin{array}{l}-1.23 \\
\end{array}$ & $\begin{array}{l}1.82 \\
213\end{array}$ & 14 & 6.29 & 0.15 & 4 & 6.28 & 0.08 & -1.23 \\
\hline $\begin{array}{l}6205 \\
6205\end{array}$ & $\begin{array}{l}11-90 \\
\text { II- } 67\end{array}$ & $\begin{array}{l}\text { SKPL } \\
\text { SKPL }\end{array}$ & $\begin{array}{l}4000 \\
3950\end{array}$ & $\begin{array}{l}0.60 \\
0.30\end{array}$ & $\begin{array}{l}-1.42 \\
-1.47\end{array}$ & $\begin{array}{l}2.13 \\
2.20\end{array}$ & $\begin{array}{l}10 \\
17\end{array}$ & $\begin{array}{l}6.11 \\
6.05\end{array}$ & $\begin{array}{l}0.09 \\
0.14\end{array}$ & $\begin{array}{l}5 \\
5\end{array}$ & $\begin{array}{l}6.16 \\
6.08\end{array}$ & $\begin{array}{l}0.14 \\
0.10\end{array}$ & $\begin{array}{l}-1.41 \\
-1.47\end{array}$ \\
\hline $\begin{array}{l}020 \\
620\end{array}$ & $\begin{array}{l}11-67 \\
\text { IV-25 }\end{array}$ & $\begin{array}{l}\text { SKPL } \\
\text { SKPL }\end{array}$ & $\begin{array}{l}3950 \\
4000\end{array}$ & 0.3 & $\begin{array}{l}-1.47 \\
-1.39\end{array}$ & 2.2 & 13 & $\begin{array}{l}.05 \\
6.14\end{array}$ & 0.14 & $\begin{array}{l}5 \\
4\end{array}$ & $\begin{array}{l}0.08 \\
6.12\end{array}$ & 0.08 & $\begin{array}{l}-1.47 \\
-1.38\end{array}$ \\
\hline & & & & & & 2 & 15 & & & 4 & & 6 & - \\
\hline 622 & 324 & $\mathrm{SK}$ & 40 & 0 & $\begin{array}{r}-1.48 \\
-1.0\end{array}$ & 2. & 18 & 6 & 0. & 4 & 6 & 15 & $\begin{array}{l}-1.48 \\
-1.48\end{array}$ \\
\hline 620 & $\mathrm{I}-4$ & SKPL & 39 & & -1 & & 16 & 6. & & 2 & & & -1.31 \\
\hline 621 & III-5 & SKPL & 4360 & 1.1 & -1.33 & 1.68 & 18 & 6.19 & 0.10 & 5 & 6.16 & 0.16 & -1.33 \\
\hline $62 \mathrm{C}$ & III-: & SK! & 4335 & & -1 & 1.9 & 17 & 6.10 & & 5 & 6.13 & 0.12 & -1.42 \\
\hline 624 & III- & SK & 42 & 0. & -1 & 2.11 & 18 & 6. & & 5 & 6.09 & 08 & -1.50 \\
\hline 628 & L 6 & & 39 & 0. & & 2 & 15 & 6. & & 4 & & 09 & -1.49 \\
\hline 620 & III- & $\mathrm{S}$ & 43 & 0 & & 2. & 16 & 6 & 1 & 3 & & 10 & -1.34 \\
\hline 620 & III-18 & SKPL & 4350 & 1. & -1.35 & 1.77 & 16 & 6.16 & 0.13 & 4 & 6.13 & 0.25 & -1.36 \\
\hline 62 & II-33 & SKPL & 4360 & 1.15 & -1.37 & 1.90 & 18 & 6.15 & 0.11 & 4 & 6.08 & 0.15 & -1.37 \\
\hline 622 & I- & SK & $42 \mathrm{~S}$ & 1. & -1.32 & 1.81 & 18 & 6.20 & 0.09 & 5 & 6.20 & 0.08 & -1.32 \\
\hline 62 & & & 41 & 0 & -1 & 1. & 18 & 6 & 0.1 & 5 & & 14 & -1 \\
\hline 620 & II-76 & SK & 43 & 1. & & 2. & 18 & 6.04 & & 5 & 6.07 & 0.11 & -1.48 \\
\hline 6205 & II -57 & & 44 & 1. & & 1.64 & 18 & 6.14 & 0.08 & 5 & 6.09 & 15 & -1.38 \\
\hline 6254 & G & SKPL & 4650 & 1.20 & -1.42 & 1.79 & 19 & 6.09 & 0.16 & 4 & 5.99 & 0.07 & -1.43 \\
\hline 6254 & II-217 & G8689 & 4410 & 1.20 & -1.42 & 1.86 & 26 & 6.10 & 0.15 & 5 & 6.27 & 0.12 & -1.42 \\
\hline
\end{tabular}

obtained applying systematic corrections to the theoretical K92 $T_{\text {eff }}$ 's, to tie them to the empirical calibrations and to the K92 colours, in order to take into account the real metallicities (different from solar). The effect of gravity was taken into account by interpolating between typical values for dwarfs and giants. The underlying assumption is that K92 models (the same consistently used in the abundance analysis) are well able to reproduce the run of colours with the overall metal content $[A / H]$, but have to be corrected in function of the surface gravity (a constant mean value of $\log g=2.75$ was assumed for all variables).

The adopted scale is very similar to the one defined by Clementini et al. (1995) to study field RR Lyrae stars; they also showed that as far as colours (and abundances) 


\begin{tabular}{|c|c|c|c|c|c|c|c|c|c|c|c|c|c|}
\hline NGC & Star & Source & Teff & $\log g$ & {$[A / H]$} & $\mathrm{Vt}$ & $\mathrm{r}$ & FeI & $\sigma(\mathrm{FeI})$ & $\mathrm{r}$ & FeII & $\sigma(\mathrm{FeII})$ & {$[\mathrm{Fe} / \mathrm{H}]$} \\
\hline 6254 & I- 15 & G8689 & 4405 & 1.00 & -1.18 & 1.45 & 24 & 6.33 & 0.13 & 2 & 6.36 & 0.02 & -1.19 \\
\hline 6254 & H-I-367 & SKPL & 4135 & 0.60 & -1.55 & 1.85 & 18 & 5.98 & 0.10 & 5 & 6.01 & 0.09 & -1.54 \\
\hline 6254 & A-III-16 & SKPL & 4150 & 0.90 & -1.38 & 1.74 & 17 & 6.15 & 0.11 & 5 & 6.07 & 0.12 & -1.37 \\
\hline 6254 & A-II-24 & SKPL & 4050 & 0.10 & -1.41 & 1.96 & 16 & 6.11 & 0.10 & 5 & 6.03 & 0.11 & -1.41 \\
\hline 6254 & A-I- 61 & SKPL & 4550 & 1.00 & -1.57 & 2.25 & 15 & 5.95 & 0.09 & 5 & 5.85 & 0.10 & -1.57 \\
\hline 6254 & H-I- 15 & SKPL & 4225 & 0.75 & -1.44 & 1.72 & 18 & 6.08 & 0.11 & 5 & 6.04 & 0.10 & -1.44 \\
\hline 6254 & A-III-5 & SKPL & 4400 & 1.20 & -1.19 & 1.63 & 18 & 6.32 & 0.10 & 4 & 6.26 & 0.12 & -1.20 \\
\hline 6254 & A-I-60 & SKPL & 4400 & 1.10 & -1.41 & 1.55 & 19 & 6.11 & 0.09 & 5 & 6.06 & 0.17 & -1.41 \\
\hline 6254 & A-III-21 & SKPL & 4060 & 0.50 & -1.39 & 2.00 & 17 & 6.13 & 0.12 & 5 & 6.06 & 0.10 & -1.39 \\
\hline 6254 & D & SKPL & 4200 & 1.05 & -1.32 & 1.78 & 16 & 6.21 & 0.11 & 5 & 6.11 & 0.12 & -1.31 \\
\hline 6254 & $\mathrm{C}$ & SKPL & 4200 & 0.75 & -1.54 & 1.84 & 18 & 5.99 & 0.07 & 5 & 5.94 & 0.05 & -1.53 \\
\hline 6254 & $\mathrm{E}$ & SKPL & 4350 & 0.80 & -1.52 & 1.99 & 18 & 6.01 & 0.10 & 5 & 5.93 & 0.03 & -1.51 \\
\hline 6254 & B & SKPL & 4150 & 0.50 & -1.40 & 1.81 & 18 & 6.12 & 0.07 & 5 & 6.03 & 0.20 & -1.40 \\
\hline 6254 & A-I-2 & SKPL & 3975 & 0.00 & -1.39 & 2.05 & 18 & 6.14 & 0.09 & 5 & 6.11 & 0.14 & -1.39 \\
\hline 6341 & XI-19 & SKPL & 4525 & 1.20 & -2.10 & 1.83 & 9 & 5.43 & 0.12 & 3 & 5.47 & 0.07 & -2.09 \\
\hline 6341 & III-82 & SKPL & 4600 & 1.47 & -2.09 & 1.75 & 7 & 5.43 & 0.13 & 2 & 5.50 & 0.22 & -2.09 \\
\hline 6341 & VII-122 & SKPL & 4350 & 0.85 & -2.15 & 1.95 & 8 & 5.36 & 0.07 & 3 & 5.27 & 0.08 & -2.16 \\
\hline 6341 & VII-18 & SKPL & 4230 & 0.70 & -2.22 & 1.99 & 10 & 5.31 & 0.09 & 5 & 5.35 & 0.07 & -2.21 \\
\hline 6341 & III-13 & SKPL & 4125 & 0.75 & -2.17 & 1.98 & 10 & 5.36 & 0.12 & 4 & 5.44 & 0.19 & -2.16 \\
\hline 6341 & II-53 & SKPL & 4370 & 0.90 & -2.11 & 1.93 & 9 & 5.42 & 0.07 & 3 & 5.37 & 0.10 & -2.10 \\
\hline 6341 & $\mathrm{~V}-45$ & SKPL & 4530 & 1.22 & -2.21 & 1.83 & 7 & 5.31 & 0.08 & 3 & 5.34 & 0.05 & -2.21 \\
\hline 6341 & XII-8 & SKPL & 4510 & 1.17 & -2.23 & 1.84 & 7 & 5.30 & 0.10 & 2 & 5.24 & 0.05 & -2.22 \\
\hline 6341 & III- 65 & SKPL & 4340 & 0.94 & -2.12 & 1.92 & 8 & 5.41 & 0.08 & 2 & 5.43 & 0.05 & -2.11 \\
\hline 6352 & 181 & G8689 & 4235 & 1.50 & -0.63 & 1.23 & 15 & 6.89 & 0.18 & & & & -0.63 \\
\hline 6352 & 111 & G8689 & 4415 & 1.80 & -0.78 & 1.88 & 19 & 6.74 & 0.19 & & & & -0.78 \\
\hline 6352 & 142 & G8689 & 4325 & 1.70 & -0.52 & 1.14 & 17 & 7.00 & 0.17 & & & & -0.52 \\
\hline 6362 & 32 & G8689 & 4210 & 1.10 & -0.98 & 1.71 & 22 & 6.55 & 0.16 & & & & -0.97 \\
\hline 6362 & 25 & G8689 & 4240 & 1.10 & -0.96 & 1.74 & 22 & 6.56 & 0.15 & & & & -0.96 \\
\hline 6397 & $\mathrm{C} 43$ & G8689 & 4526 & 1.30 & -1.64 & 1.45 & 11 & 5.88 & 0.15 & 3 & 6.19 & 0.09 & -1.64 \\
\hline 6397 & 302 & M93 & 4400 & 1.00 & -1.74 & 1.74 & 27 & 5.78 & 0.15 & 3 & 5.52 & 0.17 & -1.74 \\
\hline 6397 & C211 & NDC & 4200 & 0.70 & -1.99 & 1.84 & 46 & 5.53 & 0.08 & 7 & 5.59 & 0.13 & -1.99 \\
\hline 6397 & $\mathrm{C} 428$ & G8689 & 4669 & 1.60 & -1.68 & 1.54 & 7 & 5.83 & 0.12 & 2 & 5.97 & 0.00 & -1.69 \\
\hline 6397 & 669 & M93 & 4421 & 1.00 & -1.87 & 1.71 & 25 & 5.65 & 0.11 & 2 & 5.64 & 0.07 & -1.87 \\
\hline 6397 & 603 & M93 & 4374 & 0.90 & -1.81 & 1.62 & 23 & 5.71 & 0.13 & 2 & 5.63 & 0.05 & -1.81 \\
\hline 6397 & 468 & M93 & 4600 & 1.50 & -2.04 & 1.83 & 20 & 5.49 & 0.10 & & & & -2.03 \\
\hline 6397 & A331 & M93 & 4200 & 0.50 & -1.87 & 2.06 & 23 & 5.65 & 0.14 & & & & -1.88 \\
\hline 6397 & $\mathrm{C} 428$ & CG95 & 4669 & 1.60 & -1.73 & 1.37 & 15 & 5.79 & 0.11 & 4 & 5.88 & 0.11 & -1.73 \\
\hline 6397 & $\mathrm{C} 25$ & CG95 & 4840 & 2.00 & -1.77 & 1.51 & 8 & 5.75 & 0.12 & 2 & 5.75 & 0.16 & -1.77 \\
\hline 6397 & C211 & CG95 & 4203 & 0.70 & -1.84 & 1.88 & 19 & 5.68 & 0.09 & 6 & 5.84 & 0.13 & -1.84 \\
\hline 6397 & 469 & NDC & 4170 & 0.60 & -1.94 & 2.06 & 49 & 5.58 & 0.11 & 8 & 5.60 & 0.14 & -1.94 \\
\hline 6397 & C669 & G8689 & 4421 & 1.00 & -1.60 & 1.51 & 17 & 5.93 & 0.19 & 3 & 6.11 & 0.10 & -1.59 \\
\hline 6656 & III-52 & G8689 & 4192 & 0.80 & -1.41 & 1.90 & 25 & 6.12 & 0.15 & 5 & 6.20 & 0.15 & -1.40 \\
\hline 6656 & I-92 & G8689 & 4300 & 0.90 & -1.55 & 2.09 & 22 & 5.97 & 0.18 & 5 & 6.09 & 0.15 & -1.55 \\
\hline 6656 & III-12 & G8689 & 4189 & 0.80 & -1.49 & 2.17 & 24 & 6.03 & 0.12 & 3 & 6.16 & 0.16 & -1.49 \\
\hline 6752 & CL1015 & NDC & 4350 & 1.10 & -1.46 & 1.57 & 55 & 6.06 & 0.14 & 8 & 6.12 & 0.15 & -1.46 \\
\hline 6752 & CS3 & NDC & 4250 & 0.90 & -1.46 & 1.63 & 58 & 6.06 & 0.14 & 6 & 6.07 & 0.20 & -1.46 \\
\hline 6752 & CL1089 & NDC & 4200 & 0.80 & -1.35 & 1.85 & 59 & 6.17 & 0.11 & 8 & 6.03 & 0.10 & -1.35 \\
\hline 6752 & CL1066 & NDC & 4300 & 1.00 & -1.41 & 1.70 & 58 & 6.12 & 0.12 & 8 & 6.14 & 0.06 & -1.40 \\
\hline 6752 & A 45 & NDC & 4250 & 1.00 & -1.48 & 1.61 & 55 & 6.05 & 0.16 & 8 & 6.05 & 0.16 & -1.47 \\
\hline 6752 & 36 & M93 & 4400 & 1.00 & -1.32 & 1.58 & 29 & 6.20 & 0.11 & 3 & 6.10 & 0.10 & -1.32 \\
\hline 6752 & 284 & M93 & 4400 & 1.00 & -1.36 & 1.73 & 28 & 6.16 & 0.10 & 3 & 5.99 & 0.16 & -1.36 \\
\hline 6752 & A29 & NDC & 4350 & 1.20 & -1.45 & 1.51 & 54 & 6.07 & 0.11 & 8 & 6.14 & 0.11 & -1.45 \\
\hline 6752 & 29 & M93 & 4350 & 1.20 & -1.35 & 1.33 & 24 & 6.17 & 0.10 & 2 & 6.37 & 0.13 & -1.35 \\
\hline 6752 & A31 & CG95 & 3915 & 0.30 & -1.48 & 2.25 & 27 & 6.05 & 0.10 & 4 & 6.17 & 0.09 & -1.47 \\
\hline 6752 & $\mathrm{C} 3$ & G8689 & 4260 & 0.90 & -1.34 & 1.41 & 24 & 6.17 & 0.16 & 4 & 6.19 & 0.18 & -1.35 \\
\hline 6752 & C9 & G8689 & 4500 & 1.50 & -1.41 & 1.05 & 21 & 6.10 & 0.18 & 2 & 6.06 & 0.08 & -1.42 \\
\hline 6752 & $\mathrm{C} 118$ & G8689 & 4460 & 1.40 & -1.33 & 1.49 & 21 & 6.20 & 0.15 & 2 & 6.11 & 0.31 & -1.32 \\
\hline 6752 & A 45 & CG95 & 4258 & 1.00 & -1.41 & 1.63 & 23 & 6.11 & 0.11 & 5 & 6.17 & 0.08 & -1.41 \\
\hline 6752 & A61 & CG95 & 4310 & 1.10 & -1.61 & 2.04 & 20 & 5.91 & 0.10 & 5 & 6.12 & 0.09 & -1.61 \\
\hline 6752 & $\mathrm{C} 9$ & CG95 & 4498 & 1.50 & -1.48 & 1.89 & 15 & 6.04 & 0.14 & 3 & 6.04 & 0.07 & -1.48 \\
\hline 6809 & 283 & M93 & 4400 & 1.00 & -1.82 & 1.85 & 33 & 5.69 & 0.09 & 2 & 5.67 & 0.05 & -1.83 \\
\hline 6809 & 76 & M93 & 4400 & 1.00 & -2.10 & 1.88 & 26 & 5.42 & 0.11 & & & & -2.10 \\
\hline 6838 & A 4 & SKPL & 4100 & 0.80 & -0.59 & 1.93 & 11 & 6.93 & 0.12 & 4 & 6.78 & 0.13 & -0.59 \\
\hline 6838 & I- 45 & SKPL & 4050 & 0.80 & -0.67 & 1.82 & 13 & 6.85 & 0.12 & 2 & 6.85 & 0.03 & -0.67 \\
\hline 6838 & I- 113 & SKPL & 3950 & 0.70 & -0.72 & 1.76 & 10 & 6.81 & 0.11 & 2 & 6.70 & 0.05 & -0.71 \\
\hline 6838 & I- 46 & SKPL & 4000 & 0.80 & -0.66 & 1.89 & 10 & 6.87 & 0.15 & 2 & 6.86 & 0.17 & -0.65 \\
\hline 6838 & I & SKPL & 4200 & 1.00 & -0.87 & 1.86 & 10 & 6.65 & 0.06 & 4 & 6.49 & 0.11 & -0.87 \\
\hline 6838 & I- 53 & SKPL & 4300 & 1.40 & -0.68 & 1.78 & 16 & 6.84 & 0.13 & 4 & 6.69 & 0.08 & -0.68 \\
\hline 6838 & I- 77 & SKPL & 4100 & 0.95 & -0.67 & 1.76 & 12 & 6.86 & 0.12 & 3 & 6.89 & 0.02 & -0.66 \\
\hline 6838 & A9 & SKPL & 4200 & 1.20 & -0.83 & 1.88 & 15 & 6.70 & 0.13 & 6 & 6.64 & 0.15 & -0.82 \\
\hline 6838 & $\mathrm{~S}$ & SKPL & 4300 & 1.25 & -0.69 & 1.92 & 16 & 6.83 & 0.09 & 5 & 6.86 & 0.10 & -0.69 \\
\hline 6838 & 53 & G8689 & 4200 & 1.60 & -0.77 & 1.71 & 16 & 6.75 & 0.09 & & & & -0.77 \\
\hline 6838 & 56 & G8689 & 4580 & 2.10 & -0.51 & 1.84 & 19 & 7.01 & 0.09 & 2 & 6.68 & 0.19 & -0.51 \\
\hline 6838 & I- 21 & SKPL & 4350 & 1.45 & -0.70 & 1.93 & 16 & 6.82 & 0.15 & 5 & 6.80 & 0.10 & -0.70 \\
\hline 6838 & 21 & G8689 & 4400 & 1.65 & -0.78 & 1.51 & 16 & 6.73 & 0.11 & 1 & 6.78 & & -0.79 \\
\hline 7078 & II-75 & M93 & 4416 & 0.80 & -2.10 & 1.65 & 24 & 5.41 & 0.16 & 1 & 5.07 & & -2.11 \\
\hline 7078 & II-75 & SKPL & 4410 & 0.90 & -2.11 & 1.93 & 8 & 5.41 & 0.12 & 2 & 5.03 & 0.12 & -2.11 \\
\hline 7078 & $\mathrm{~s} 6$ & M93 & 4460 & 1.00 & -2.11 & 1.56 & 22 & 5.40 & 0.21 & 3 & 5.49 & 0.15 & -2.12 \\
\hline 7078 & IV-38 & SKPL & 4300 & 0.60 & -2.13 & 2.03 & 8 & 5.38 & 0.20 & 1 & 5.26 & & -2.14 \\
\hline 7078 & $\mathrm{~S} 1$ & SKPL & 4410 & 0.90 & -2.13 & 1.93 & 8 & 5.39 & 0.09 & 1 & 5.45 & & -2.13 \\
\hline 7099 & D & M93 & 4600 & 1.50 & -1.91 & 1.39 & 24 & 5.61 & 0.28 & 2 & 5.58 & 0.16 & -1.91 \\
\hline 7099 & 157 & M93 & 4600 & 1.50 & -1.90 & 1.30 & 21 & 5.62 & 0.17 & 3 & 5.32 & 0.11 & -1.90 \\
\hline
\end{tabular}

are concerned, K92 models are well suited to reproduce the atmospheres of RR Lyrae variables. ${ }^{11}$

11 The reader should be aware of the small inconsistency due to our use of the CFP scale for globular cluster (cooler) giants and the new temperature scale for RR Lyraes; however, as discussed in Gratton et al. (1996), differences between the two scales are well within the intrinsic uncertainties of both scales and do not alter significatively our conclusions.
The photometry for the variables of M 15 is taken from the high quality work of Bingham et al. (1984). For M 3, we used colours from Sandage (1990). The choice of the mean colour which better represents the one the variable should have were it not pulsating is not simple (see e.g., Fernley 1994). However, for sake of comparison with Sandage's previous works on M 3 , in the present study we use $(<B>-<V>)_{\text {corr }}$, the corrected colours taken 


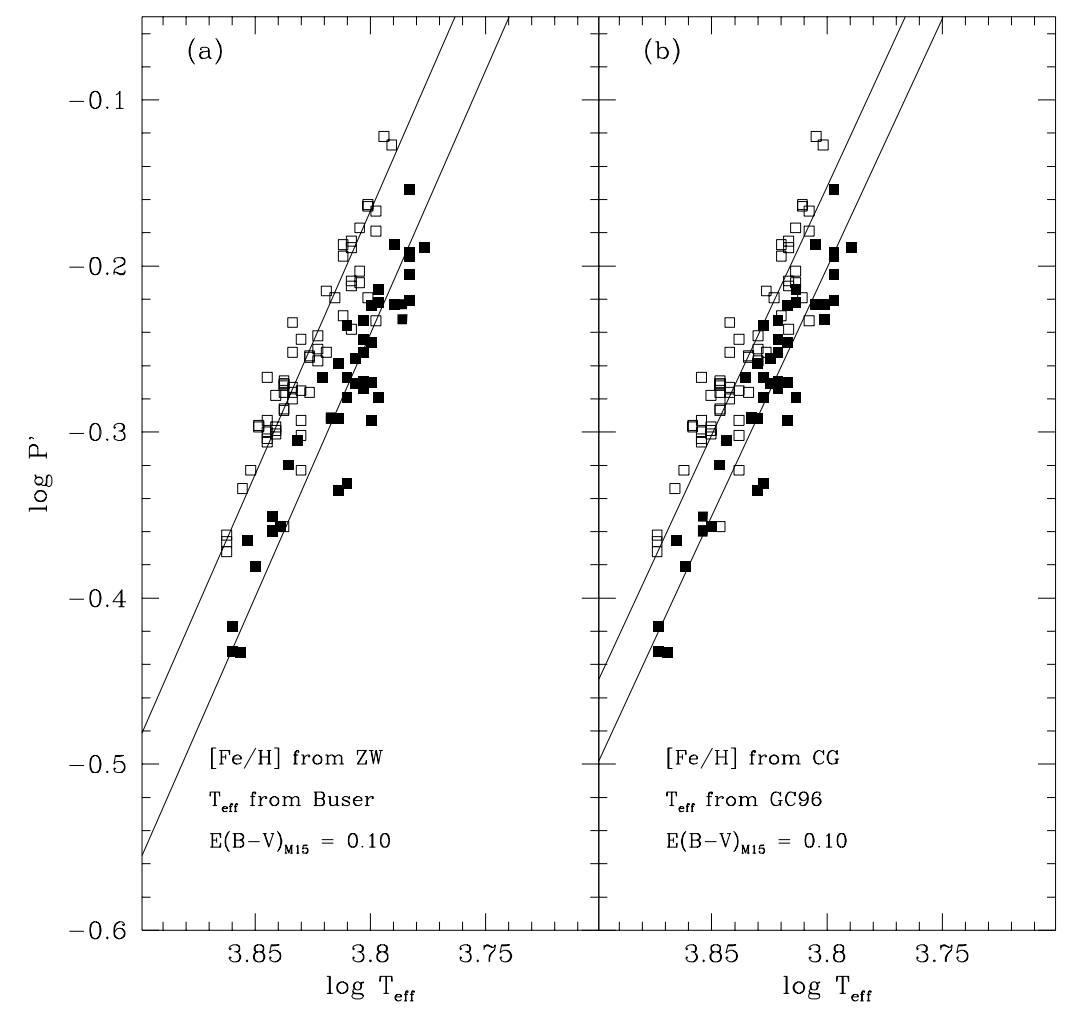

Fig. 7. Observed distributions of variables in M 3 (filled squares) and M 15 (open squares) in the $\log P^{\prime}-\log T_{\text {eff }}$ plane (see text)

from the quoted sources; they also include an empirical correction $\Delta C(\mathrm{~A})$ depending on the light-curve amplitude (Sandage 1990).

We adopt $E(B-V)=0.00$ for M 3, while for M 15 we assume 0.10 (Zinn 1980).

Figure 7 shows the observed distributions of variables in M 3 and M 15 in the classical $\log P^{\prime}-\log T_{\text {eff }}$ plane $\left(\log P^{\prime}\right.$ is the reduced and fundamentalized period; for ctype RR Lyraes the period is fundamentalized by adding 0.127 to $\log \mathrm{P})$. The lines are best-fits drawn by eye through the data, since the computed least squares linear regression result in non-parallel lines, due to the scatter among the data, especially in those of M 3. This method is good enough in this case, since we are only interested in presenting and emphasizing another source of uncertainty affecting this method.

In panel (a) $\mathrm{T}_{\text {eff's }}$ are derived from the old Kurucz (1979) model atmospheres, following the (unpublished) calibration of Buser, and with $E(B-V)=0.00$ and 0.10 for M 3 and M 15 respectively. Metallicities are on the Zinn and West's scale: this panel should then reproduce closely enough Sandage's results for the Oosterhoff effect in the pair M 3-M 15. In panel (b) we used the new metallicities from the present work and $T_{\text {eff }}$ 's derived from K92 models.

In order to evaluate the period shift between the two distributions, we followed the prescriptions of Sandage (1993b), reading the values of $\log P^{\prime}$ not at constant $\log T_{\text {eff }}$, but at lower temperatures for lower metallicities, following his Eq. (5). Our measurements then give the period shift $\Delta \log P$ (in the sense M $15-\mathrm{M} 3)=-0.103$ and -0.076 for case (a) and (b), respectively. We note that only the first slope is similar to the one derived by Sandage (1993a: $\Delta \log P / \Delta[\mathrm{Fe} / \mathrm{H}]=-0.122)$. Simply using the new metallicities and effective temperatures, although derived from the same photometric data, significatively decreases the size of the effect.

Taking at face value these figures, and using the parameters temperature and mass (along with their variations with metallicity as given by Sandage 1993b) in the pulsation equation of van Albada \& Baker (1973), we obtain for the slope of the relationship $M_{\text {bol }}$ vs. $[\mathrm{Fe} / \mathrm{H}]$ the values 0.300 and 0.221 for case (a) and (b) respectively. As one can see, case (b) is very similar to that derived from the Baade-Wesselink analyses (0.25, as quoted for instance in Sandage 1993b), whose results were considered up to now in serious disagreement with the calibration from the Oosterhoff effect.

This straightforward exercise points out that simply using new, modern, and self-consistent temperatures and metallicities, the size of the Oosterhoff effect in the template pair M 3-M 15 is somewhat reduced, being more consistent with theoretical models of Zero Age Horizontal Branch (see Sandage 1993b for references). We then 
conclude that our effort in obtaining these new, improved values for metallicities can pay off; we will proceed to a new calibration of photometric / low resolution indices, including all known globular clusters, as soon as a homogeneous dataset will be available.

Acknowledgements. We wish to thank Chris Sneden for useful discussion and encouragement. E.C. warmly thanks Carla Cacciari for providing tables with the parameters of variables in M 3 and M 15 from Kurucz's (1979) models and for many useful suggestions and discussions on the Oosterhoff effect. E.C. acknowledges financial support from the Consiglio Nazionale delle Ricerche.

\section{References}

Anders E., Grevesse N., 1989, Geochim. Cosmochim. Acta 53, 197

Armandroff T.E., Zinn R., 1988, AJ 96, 92

Arp H.C., 1955, AJ 60, 317

Bard A., Kock A., Kock M., 1991, A\&A 248, 315

Bard A., Kock M., 1994, A\&A 282, 1014

Bell R.A., Gustafsson B., 1989, MNRAS 236, 653

Bell R.A., Eriksson K., Gustafsson B., Nordlund A., 1976, A\&AS 23, 37 (BEGN)

Biémont E., Baudoux M., Kurucz R.L., Ansbacher W., Pinnington E.H., 1991, A\&A 249, 539

Bingham E.A., Cacciari C., Dickens R.J., Fusi Pecci F., 1984, MNRAS 209, 765

Blackwell D.E., Lynas-Gray A.E., 1994, A\&A 282, 899

Blackwell D.E., Shallis M.J., Simmons G.J., 1980, A\&A 81, 340

Butler D., 1975, ApJ 200, 68

Carbon D., Langer G., Butler D., Kraft R., Suntzeff N., Kemper E., Trefzger C., Romanishin W., 1982, ApJS 49, 207

Carretta E., Gratton R.G., Sneden C., 1996 (in preparation)

Castelli F., Gratton R.G., Kurucz R.L., 1996 (submitted to A\&A)

Clementini G., Carretta E., Gratton R.G., Merighi R., Mould J.R., McCarthy J.K., 1995, AJ 110, 2319

Cohen J.G., 1983, ApJ 270, 654

Cohen J., Frogel J.A., Persson S.E., 1978, ApJ 222, 165 (CFP)

Costar D., Smith H.A., 1988, AJ 96, 1925

Dalle Ore C., 1992, Ph.D. Thesis, Un. California

Dalle Ore C., Gratton R.G., Peterson R., 1996 (in preparation)

Di Benedetto G.P., Rabbia Y., 1987, A\&A 188, 114

Djorgovski S., 1993, In Structure and Dynamics of Globular Clusters, Djorgovski S.G. and Meylan G. (eds.), ASP Conf. Ser. 50 , p. 373

Fernley J., 1994, A\&A 268, 591

Frogel J.A., Persson S.E., Cohen J.G., 1979, ApJ 227, 499

Frogel J.A., Persson S.E., Cohen J.G., 1981, ApJ 246, 842
Frogel J.A., Persson S.E., Cohen J.G., 1983, ApJS 53, 713

Gratton R.G., 1987, A\&A 179, 181 (G87)

Gratton R.G., 1988, Rome Obs. Preprint Ser. 29

Gratton R.G., Carretta E., Castelli F., 1996, A\&A (in press)

Gratton R.G., Ortolani S., 1989, A\&A 211, 41 (G89)

Gratton R.G., Sneden C., 1994, A\&A 287, 927

Gratton R.G., Quarta M.L., Ortolani S., 1986, A\&A 169, 208 (G86)

Hannaford P., Lowe R.M., Grevesse N., Noels A., 1992, A\&A 259,301

Heise C., Kock M., 1990, A\&A 230, 244

Holweger H., Müller E.A., 1974, Solar Phys. 39, 19

Kraft R.P., 1994, PASP 106, 553

Kraft R.P., Sneden C., Langer G.E., Prosser C.F., 1992, AJ 104, 645 (SKPL2)

Kraft R.P., Sneden C., Langer G.E., Shetrone M.D., 1993, AJ 106, 1490 (SKPL4)

Kraft R.P., Sneden C., Langer G.E., Shetrone M.D., Bolte M., 1995, AJ 109, 2586 (SKPL6)

Kurucz R.L., 1992 (private communication) (K92)

Kurucz R.L., 1979, ApJS 40, 1

Leep E.M., Oke J.B., Wallerstein G., 1987, AJ 93, 338

Mäckle R., Griffin R., Griffin R., Holweger H., 1975, A\&AS 19, 303

Magain P., 1984, A\&A 134, 189

Manduca A., 1981, ApJ 245, 258

McWilliam A., Geisler D., Rich R.M., 1992, PASP 104, 1193

Minniti D., Geisler D., Peterson R.C., Claria J.J., 1993, ApJ 413,548

Norris J., Da Costa G., 1995, ApJ 447, 680

Oosterhoff P., 1944, BAN 10, 55

Pilachowski C., Sneden C., Wallerstein G., 1983, ApJS 52, 241

Preston G.W., 1959, ApJ 130, 507

Rutten R.J., van der Zalm E.B.J., 1984, A\&AS 55, 143

Sandage A., 1990, ApJ 350, 603

Sandage A., 1993a, AJ 106, 703

Sandage A., 1993b, AJ 106, 719

Simmons G.J., Blackwell D.E., 1982, A\&A 112, 209

Smith H.A., 1984, ApJ 281, 148

Smith H.A., 1987, PASP 94, 122

Sneden C., Kraft R.P., Prosser C.F., Langer G.E., 1991, AJ 102, 2001 (SKPL1)

Sneden C., Kraft R.P., Prosser C.F., Langer G.E., 1992, AJ 104, 2121 (SKPL3)

Sneden C., Kraft R.P., Langer G.E., Prosser C.F., Shetrone M.D., 1994, AJ 107, 1773 (SKPL5)

Trefzger C., Carbon D., Langer G., Suntzeff N., Kraft R., 1983, ApJ 266, 144

Tucholke H.-J., 1992, A\&AS 93, 293

van Albada T.S., Baker N., 1973, ApJ 185, 477

Zinn R., 1985, ApJ 293, 424

Zinn R., 1980, ApJS 42, 19

Zinn R., West M.J., 1984, ApJS 55, 45 (ZW) 